\title{
European guidelines for quality assurance in colorectal cancer screening and diagnosis. First Edition Faecal occult blood testing
}

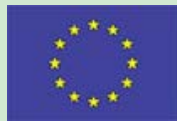

Co-Funded by the Health Programme of the European Union

Authors

Institutions

\author{
S. P. Halloran' ${ }^{1}$ G. Launoy ${ }^{2}$, M. Zappa ${ }^{3}$
}

${ }^{1}$ Bowel Cancer Screening Southern Programme Hub, Royal Surrey County Hospital NHS Foundation Trust and University of Surrey, Guildford, United Kingdom

INSERM, Caen University, Caen, France

Cancer Prevention and Research Institute (ISPO), Florence, Italy
Keywords

mass screening

- colorectal neoplasms

stool test

- laboratory quality control

- quality assessment

- multidisciplinary evidencebased guidelines

- population-based programmes
Bibliography

DOI http://dx.doi.org/

10.1055/s-0032-1309791

Endoscopy 2012; 44:

SE65-SE87

(c) Georg Thieme Verlag KG Stuttgart · New York

ISSN 0013-726X

\section{Corresponding author}

\section{S. P. Halloran}

Royal Surrey County Hospital NHS Foundation Trust

Egerton Road

Guildford, Surrey

GU2 7XX

United Kingdom.

s.halloran@nhs.net

qas@iarc.fr
Multidisciplinary, evidence-based guidelines for quality assurance in colorectal cancer screening and diagnosis have been developed by experts in a project coordinated by the International Agency for Research on Cancer. The full guideline document covers the entire process of populationbased screening. It consists of 10 chapters and over 250 recommendations, graded according to the strength of the recommendation and the supporting evidence. The 450-page guidelines and the extensive evidence base have been published by the European Commission. The chapter on fae-

\section{Background \\ $\nabla$}

According to the most recent estimates by the International Agency for Research on Cancer [33] colorectal cancer (CRC) is the most common cancer in Europe with 432000 new cases in men and women reported annually. It is the second most common cause of cancer deaths in Europe with 212000 deaths reported in 2008. Worldwide CRC ranks third in incidence and fourth in mortality with an estimated 1.2 million cases and 0.6 million deaths annually. The European Union (EU) recommends population-based screening for breast, cervical and colorectal cancer using evidence-based tests with quality assurance of the entire screening process including diagnosis and management of patients with screen-detected lesions [25]. The EU policy takes into account the principles of cancer screening developed by the World Health Organization [128] and the extensive experience in the EU in piloting and implementing population-based cancer screening programmes [123]. Screening is an important tool in cancer control in countries with a significant burden of CRC, provided the screening services are high quality [124]. The presently reported multidisciplinary, evidence-based guidelines for quality assurance in colorectal cancer screening and cal occult blood testing includes 21 graded recommendations. The content of the chapter is presented here to promote international discussion and collaboration by making the principles and standards recommended in the new EU Guidelines known to a wider professional and scientific community. Following these recommendations has the potential to enhance the control of colorectal cancer through improvement in the quality and effectiveness of screening programmes and services.

diagnosis have been developed by experts and published by the EU [107].

\section{Methods \\ $\nabla$}

The methods used are described in detail elsewhere in this supplement [79]. Briefly, a multidisciplinary group of authors and editors experienced in programme implementation and quality assurance in colorectal cancer screening and in guideline development collaborated with a literature group consisting of epidemiologists with special expertise in the field of CRC and in performing systematic literature reviews. The literature group systematically retrieved, evaluated and synthesized relevant publications according to defined clinical questions (modified PatientIntervention-Comparison-Outcome-Study method). Bibliographic searches for most clinical questions were limited to the years 2000 to 2008 and were performed on Medline, and in many cases also on Embase and The Cochrane Library. Additional searches were conducted without date restrictions or starting before 2000 if the authors or editors who were experts in the field knew that there were relevant articles published before 2000. Articles of adequate quality recommended 
by authors because of their clinical relevance were also included. Only scientific publications in English, Italian, French and Spanish were included. Priority was given to recently published, systematic reviews or clinical guidelines. If systematic reviews of high methodological quality were retrieved, the search for primary studies was limited to those published after the last search date of the most recently published systematic review, i.e. if the systematic review had searched primary studies until February 2006, primary studies published after February 2006 were sought. If no systematic reviews were found, a search for primary studies published since 2000 was performed.

In selected cases references not identified by the above process were included in the evidence base, i.e. when authors of the chapters found relevant articles published after 2008 during the period when chapter manuscripts were drafted and revised prior to publication. The criteria for relevance were: articles concerning new and emerging technologies where the research grows rapidly, high-quality and updated systematic reviews, and large trials giving high contribution to the robustness of the results or allowing upgrading of the level of evidence.

The methodological quality of the retrieved publications was assessed using the criteria obtained from published and validated check lists. Evidence tables were prepared for the selected studies. The evidence tables, clinical questions and bibliographic literature searches are documented elsewhere [78].

In the full guidelines document prepared by the authors and editors [107] over 250 recommendations were formulated according to the level of the evidence and the strength of the recommendation using the following grading scales.

\section{Level of evidence:}

I multiple randomised controlled trials (RCTs) of reasonable sample size, or systematic reviews (SRs) of RCTs

II one RCT of reasonable sample size, or 3 or less RCTs with small sample size

III prospective or retrospective cohort studies or SRs of cohort studies; diagnostic cross-sectional accuracy studies

IV retrospective case-control studies or SRs of case-control studies, time-series analyses

V case series; before/after studies without control group, crosssectional surveys

VI expert opinion

\section{Strength of recommendation:}

A intervention strongly recommended for all patients or targeted individuals

B intervention recommended

C intervention to be considered but with uncertainty about its impact

D intervention not recommended

E intervention strongly not recommended

Some statements of advisory character considered to be good practice but not sufficiently important to warrant formal grading were included in the text.

\section{Results}

$\nabla$

Twenty-one graded recommendations are provided in Chapter 4.

\section{Recommendations ${ }^{1}$}

$\nabla$

\section{Guaiac faecal occult blood tests (gFOBT)}

4.1 Guaiac faecal occult blood tests (gFOBT) have proven characteristics that make them suitable for population screening. They lack the analytical specificity and sensitivity of immunochemical tests, their analysis cannot be automated and the concentration at which they turn from negative to positive cannot be adjusted by the user. For these reasons guaiac-based tests are not the preferred test for a modern population screening programme, although depending on local labour costs, the mechanism of kit distribution and collection and reduced sample stability in immunochemical testing, they might prove more practicable and affordable than immunochemical testing (I-B). ${ }^{\text {Sect 4.2.4; }}$ 4.2.7; $4.3 ; 4.4 .2$

\section{Faecal immunochemical tests for haemoglobin (FIT)}

4.2 Immunochemical tests have improved test characteristics compared with conventional guaiac-based tests. They are analytically and clinically more sensitive and specific, their measurement can be automated and the user can adjust the concentration at which a positive result is reported. Immunochemical tests are currently the test of choice for population screening; however, individual device characteristics including ease of use by the participant and laboratory, suitability for transport, sampling reproducibility and sample stability are all important when selecting the FIT most appropriate for an individual screening programme (II-A). ${ }^{\text {Sect } 4.2 .5 ; ~ 4.2 .7 ; ~ 4.3 ; ~ 4.4 .2 ~}$

\section{DNA and other related new markers}

4.3 Only tests for blood in faeces have been demonstrated to have the necessary characteristics to be suitable for population screening. DNA and other related new markers are currently unsuitable for screening, either singly or as members of a panel of tests (III-D). ${ }^{\text {Sect } 4.2 .6 ; 4.2 .7}$

\section{Sample stability between collection and analysis}

4.4 Whilst a maximum period of 14 days between collection and analysis is quoted for many guaiac faecal occult blood tests, that quoted for immunochemical tests is significantly shorter. Until more stability data are published, screening programmes should adopt the conditions and period of storage described in manufacturer's Instructions for Use having determined that they are appropriate for local conditions which might expose samples to high temperatures for long periods of time (III-A). ${ }^{\text {Sect 4.3.3.2; 4.3.4 }}$

\section{Screening algorithm}

\section{- Sample and test numbers}

4.5 Few studies have examined the number of stool specimens necessary to optimise the diagnostic performance of FOBT. Consideration should be given to using more than one spe-

\footnotetext{
${ }^{1}$ Sect (superscript) after each recommendation in the list refers the reader to the section/s of the Guidelines dealing with the respective recommendation.*

$\operatorname{Rec}$ (superscript) throughout the chapter refers to the number of the recommendation dealt with in the preceding text.*

* The first digit of the section numbers and recommendation numbers refers to the respective chapter in the guidelines. For Chapters 1 to 3 see: $[65,76$, $84]$ and for Chapters 5 to 10 see: $[118,115,92,114,7,8]$, respectively.
} 
cimen together with criteria for assigning positivity to provide a referral rate that is clinically, logistically and financially appropriate to the screening programme. The clinical sensitivity and specificity of testing can be modified depending on how the test data are used. Guaiacbased tests typically use 3 stools, but an algorithm using additional tests can be used to adjust clinical sensitivity and specificity (III-C). ${ }^{\text {Sect 4.4.3.2; 4.4.3.1; 4.4.4 }}$

\section{- Determining test positivity}

4.6 The choice of a cut-off concentration to be used in an immunochemical test to discriminate between a positive and negative result will depend on the test device chosen, the number of samples used and the algorithm adopted to integrate the individual test results. Whilst an increasing number of studies are reporting the experience of different algorithms, local conditions, including the effect on sample stability of transport conditions, preclude a simple prescribed algorithm at this time. Adoption of a test device and the selection of a cut-off concentration should follow a local pilot study to ensure that the chosen test, test algorithm and transport arrangements work together to provide a positivity rate that is clinically, logistically and financially acceptable (VI-A). Sect 4.4.3.1; 4.4.3.2; 4.4.4

\section{Test interference}

\section{- Dietary restriction}

4.7 Dietary constituents present potential interference in guaiac faecal occult blood tests. Dietary restriction has not been demonstrated to significantly increase screening specificity, and risks reducing participation rate. The potential for dietary interference is significantly less for immunochemical tests. With the qualification that a diet peculiar to a particular country or culture may not have been tested or reported, dietary restriction is not indicated for programmes using either guaiac-based or immunochemical tests (II-D). ${ }^{\text {Sect 4.3.2.1; 4.3.2.3; 4.3.4 }}$

\section{- Drug restriction}

4.8 Interference from bleeding associated with drugs such as aspirin, nonsteroidal anti-inflammatory drugs and anticoagulants (e.g. warfarin) present potential interference in both guaiac and faecal immunochemical tests for haemoglobin. Although the literature carries some contradictory reports of the effect of anticoagulants on screening outcome, drug restriction is not recommended for population screening programmes using either guaiac-based or immunochemical tests (III-D). . Sect 4.3.2.2; 4.3.2.3; 4.3.4

\section{Faecal sampling/collection system}

4.9 Many factors influence the uptake and reliability of sample collection. Inappropriate implementation can result in grossly misleading results. No single collection method is supported by the literature; however, the following factors should be considered when selecting a device for taking samples in population screening:

- The distribution process should be reliable and reach all selected subjects.

- The laboratory should be able to identify the subject ID on the test device unambiguously, perhaps using a suitable barcode.

- The laboratory should be able to check the manufacturer's device expiry date on each returned device.
- The instructions for using the device must be simple and clear.

- The device should to be simple and easy to use by the target population.

- The device should leave minimal opportunity for collection error.

- The device should facilitate consistency in the volume of sample collected.

- The device/instructions should discourage inappropriate repeat sampling into/onto the sample device.

- Misuse of the device by participants should not cause loss of sample buffer.

- The system should not be susceptible to interference from toilet bowl disinfectants, etc.

- The screening participant should be able to record the date of sample collection to ensure the laboratory can verify receipt within an acceptable sample stability period.

- The process used by the subject for returning the device should be simple, reliable, safe and, when appropriate, should meet EU postal regulations.

A local pilot study should be undertaken to ensure that the chosen device and associated distribution, sampling and la-

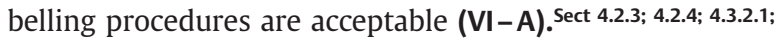
4.3.3.4; 4.3.4

\section{Laboratory organisation}

\section{- Number of laboratory sites}

4.10 Population screening necessitates the receipt, measurement and recording of thousands of tests each day. The samples should be analysed without delay to avoid further sample degradation and avoid an increase in false negative results. Interlaboratory analytical imprecision is well described and can be observed through established external quality assurance schemes. Improved consistency is achieved by adopting common analytical platforms, analytical and quality standards and shared staff training. The analysis needs to be reproducible across a screening population and therefore the number of analytical centres should be minimised with automated analytical systems utilised wherever possible and agreed common testing procedures adopted by each centre (VI-B). ${ }^{\text {sect 4.3.3.4; 4.3.4 }}$

\section{- Laboratory staff}

4.11 All laboratories providing population screening should be led by a qualified clinical chemist who is trained and experienced in the techniques used for analysis and with clinical quality assurance procedures (VI-B). . $^{\text {sect 4.3.3.4; 4.3.4 }}$

- Laboratory accreditation and quality monitoring

4.12 All laboratories providing screening services should be associated with a laboratory accredited to ISO 15189:2007 Medical laboratories - Particular requirements for quality and competence (http://www.iso.org/iso/iso_catalogue.htm). The laboratories should perform internal quality control (IQC) procedures and participate in an appropriate external quality assessment scheme (EQAS) (VI-B). Sect 4.3.3.4

Distribution of FOBT kits by mail

4.13 Distribution and receipt of FOBT kits using local postal services can be an effective means of reaching the designated population (Ch. 2 [76], Rec. 2.15) (II-B). Sect 2.5.1.1; 4.2.1; 4.3.3.4 
Maximisation of uptake - Influencing factors associated with the test kit

4.14 The choice of the test kit must be influenced by factors that enhance accessibility and uptake (see below and Ch. 2 [76], Rec. 2.14) (II-A): Sect 4.2.3; 4.2.4; 4.4.4

- Dietary restrictions

In order to enhance participation in screening, test kits should not require dietary restrictions (Ch. 2 [76], Rec. 2.17) (II-A). Sect 4.3.2.1; 4.3.2.3; 4.4.4; 2.5.1.2

- Kit design

The design of a test kit should make it acceptable to the target population (see Ch. 2 [76], Rec. 2.14) (II-A). Sect 4.2.3; 4.2.4; 4.4.4; 2.5.1.1

- Simple and clear instructions

A clear and simple instruction sheet should be provided

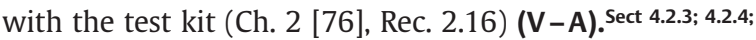
4.4.4; 2.5.1.1

\section{Identification of participants and test results}

4.15 Automated check protocols should be implemented to ensure correct identification of the screened population and complete and accurate recording of individual screening participation and test results (see Ch. 2 [76], Rec. 2.18) (VI-A). Sect 4.3.4; 2.5.1.3

\section{Classification of test results}

4.16 Protocols should be implemented to ensure standardised and reliable classification of the test results (Ch. 2 [76], $\operatorname{Rec} 2.19)(\mathrm{VI}-\mathrm{A})$. Sect 4.3.4; 2.5.1.3

\section{Quality Assurance}

\section{- Quality assurance of gFOBT testing}

4.17 Whilst an immunochemical test is recommended, programmes that adopt a traditional guaiac test need to apply additional laboratory quality procedures. To minimise variability and error associated with visual test reading, including manual results input, the following procedures should be considered ( $\mathrm{VI}-\mathrm{B})$ : ${ }^{\text {Sect }}$ 4.3.3.4; 4.3.4

- Use of appropriate temperature, artificial lighting and neutral-coloured walls in the reading laboratory;

- Use of a national laboratory training programme to prosper consistency of interpretation;

- A blinded internal QC check each day for each analyst before commencing testing;

- Adoption of a monitoring programme to identify operator-related analytical performance (e.g. positivity variability and bias); and

$\checkmark$ Double entry of test results

\section{- Quality assurance of FIT testing}

4.18 Consistency in analytical performance must be assured by the adoption and application of rigorous quality assurance procedures. Manufacturer's instructions for use must be followed. Laboratories should perform daily checks of analytical accuracy and precision across the measurement range with particular emphasis at the selected cut-off limit. Rigorous procedures need to be agreed and adopted on how internal quality control data are interpreted and how the laboratory responds to unsatisfactory results. Performance data, both internal quality control and external quality assessment data, should be shared and reviewed by a quality assurance team working across the programme. Sufficient instrumentation should be available to avoid de- lays in analysis due to instrument failure or maintenance procedures (VI-B). ${ }^{\text {Sect 4.3.3.4; } 4.3 .4}$

\section{- External quality assessment}

4.19 A European external quality assessment scheme should be developed to facilitate Europe-wide quality assurance of occult blood testing and enhance the reproducibility of testing within and between countries providing population screening (III-B). Sect 4.3.3.4; 4.3.4

\section{- Outcome monitoring}

4.20 All aspects of laboratory performance in respect of the screening test should be part of a rigorous quality assurance system. Uptake, undelivered mail, time from collection to analysis, analytical performance (internal QC and external QA), positivity rates, lost \& spoilt kits and technical failure rate, technician performance variability and bias should each be subject to rigorous monitoring $(\mathbf{V I}-\mathbf{A})$. . $^{\text {sect }}$ 4.3.3.4; 4.3.4

\section{- Quality of information}

4.21 The proportion of unacceptable tests received for measurement is influenced by the ease of use of the test kit and the quality of the instructions for use. This proportion should not exceed $3 \%$ of all kits received; less than $1 \%$ is desirable (see Ch. 3 [84], Rec. 3.9) (III-A). Sect 4.3.4; 3.3.2

\subsection{Introduction}

\section{$\nabla$}

The ideal biochemical test for population-screening of colorectal cancer would use a biomarker, specific and sensitive for both cancer and pre-cancer, on an easily collected sample, which could be safely and cheaply transported to a centralised laboratory for accurate, reproducible, and cheap automated analysis. None of the currently available tests fully meet all of those criteria.

That colorectal cancers and adenomatous polyps bleed, be it to varying degrees and perhaps intermittently, has provided faecal blood haemoglobin as the biomarker of choice for current screening programmes. The presence of blood in faeces can be due to pathological conditions other than neoplasia, from physiological blood loss of between 0.5 and $1.0 \mathrm{~mL} / \mathrm{d}$ [80], from vigorous brushing of gums and from dietary constituents such as meat and meat products [34].

The cheapest but least specific means of detecting haemoglobin uses guaiac gum, is often referred to as the guaiac faecal occult blood test or gFOBT, and its efficacy as a colorectal cancer screening test has been demonstrated in three randomised controlled trials $[48,63,77]$. The test detects the haem component of haemoglobin, which is identical across human and animal species and is chemically robust and only partially degraded during its passage through the gastrointestinal tract. gFOBTs provide little specificity for lesions of the distal intestinal tract and cannot distinguish between human blood and blood residues from the diet. The analytical sensitivity of gFOBTs to haemoglobin can be increased by hydrating the sample before analysis; however this brings little benefit because increased clinical sensitivity is accompanied by decreased clinical specificity. More subtle adjustment to the analytical sensitivity of gFOBTs is not technically possible, and screening programmes must configure their programme algorithm (the required number of stool samples and the required number of positive test spots) and secondary investigations, usually colonoscopy, to meet the gFOBT positivity rate. 
A significant technical enhancement to the simple guaiac test for blood is achieved by using an antibody (immunoglobulin) specific to human globin, the protein component of haemoglobin. These immunochemical techniques use specific antibodies and are well-established and ubiquitous in clinical laboratories. At the point-of-care, immunochemical tests have been widely adopted, notably in fertility, pregnancy and drug tests.

Whilst the haem component of blood is common to all species, globin is conveniently species-specific, so faecal immunochemical tests for haemoglobin, or FIT should not be subject to interference from dietary blood. Detection of globin also confers the advantage of making the test more specific to bleeding from the distal gastrointestinal tract, since protease enzymes gradually digest blood from the proximal tract during its passage through the intestine, rendering it less likely to be recognised by the antibodies used in an FIT.

Immunochemical technology enables detection of blood at lower concentrations than gFOBTs and therefore increases clinical sensitivity by detecting smaller blood losses from small or intermittently bleeding lesions. Whilst improved analytical specificity reduces false-positive tests from dietary blood, their increased analytical sensitivity means that small losses from inflammatory diseases or physiological sources will bring new false-positives with a higher positivity rate and decreased specificity. Several newer FIT have the ability to adjust and set the cut-off concentration above which the device will report a positive result. These adjustments are made on an instrument reader, and such instruments can provide the additional and important opportunity of automating the process. Examples of products with these characteristics are the OC-Sensor Diana, Eiken Chemical Co., Tokyo, Japan, and the SENTiFOB, Sentinel Diagnostics SpA, Milan, Italy.

Population screening for colorectal cancer can now benefit from tests that have an adjustable detection limit and have the efficiencies and analytical reproducibility facilitated through automated analysis; currently only FIT provides this opportunity.

\subsection{Biochemical tests for colorectal cancer}

\section{$\nabla$}

\subsubsection{Characteristics of a test for population-screening of} colorectal cancer

The list below summarises the analytical and clinical aspects of biomarker testing that make it suitable for population screening and identifies characteristics that are important for effective and efficient implementation.

Testing Process

a) Sample

I. Reliable sample collection, reproducible sample size

II. Sample collection process is simple requiring minimal contact with the stool

III. Safe and acceptable for the chosen method of transport, meets EU mail regulations

b) Biomarker (analyte)

I. Sufficiently stable, at ambient temperature, between sample collection and testing

II. Analytical sensitivity and specificity

1. Adequate analytical sensitivity and specificity

2. Adequate discrimination between neoplastic colorectal pathology and other pathologies or physiological sources of the biomarker

3. Minimal analytical or biological interference (e.g. diet and drugs)
III. Ability to adjust sensitivity (and specificity) to be clinically and practically acceptable

c) Analysis

I. Easy and reliable to measure

II. Amenable to automation

III. Acceptably reproducible

IV. Amenable to quality control and assessment monitoring

d) Availability of test

I. Reliable commercial source, long-term quality provider

II. Acceptable inter and intra-batch reproducibility

III. Affordable

Clinical Outcome

a) Acceptable clinical performance

I. Sensitivity

II. Specificity

III. Positive predictive value

The outcome of a screening test must be the identification of an acceptable proportion of the population who have early-stage colorectal cancer or adenoma and are amenable to successful treatment [128]. The screening test must also show adequate discrimination between those who have the disease and those who do not. Critically, the clinical sensitivity and specificity of the test and the way it is implemented must identify only the number of participants that is logistically and financially acceptable for referral to colonoscopy clinics.

When interpreting the clinical sensitivity and specificity of tests described in the literature, it is important to do so in the specific context of the study, the method of implementation, the nature of the population served and other local health and social welfare issues.

\subsubsection{Faecal blood loss}

An abnormal increase in blood loss into the intestine is necessary for the success of gFOBTs and FIT. Faecal haem, haem-derived porphyrin and 51-chromium-labelled red cells have all been used to determine physiological blood loss. A recent systematic review by Moore, Derry \& McQuay [80] of the effect of non-steroidal anti-inflammatory drugs (NSAIDs) on blood loss showed a normal daily loss in 1000 participants of less than $1 \mathrm{~mL} / \mathrm{d}$. Blood losses greater than $1 \mathrm{~mL} / \mathrm{d}$ may be seen following vigorous brushing of teeth and gums, and in irritation and inflammation of the intestinal tract. Most NSAIDs, and aspirin in low doses, produce an increased blood loss of 1 to $2 \mathrm{~mL} / \mathrm{d}$, which increases to $5 \mathrm{~mL} / \mathrm{d}$ in $5 \%$ and $10 \mathrm{~mL} / \mathrm{d}$ or more in $1 \%$ of those taking larger doses. Large daily aspirin doses of $1800 \mathrm{mg}$ or more, cause daily blood losses of between $5 \mathrm{~mL} / \mathrm{d}$ and $10 \mathrm{~mL} / \mathrm{d}$. Other chronic inflammatory conditions of the gastrointestinal tract including inflammatory bowel disease, colitis, Crohn's disease and perianal lesions also increase blood loss.

Macrae \& St. John [75] showed the close relationship between adenoma size and blood loss using 51-chromium-labelled red cells. Levi et al. [68] used the FIT OC-Sensor to show increasing faecal haemoglobin from normal and hyperplastic polyps through non-advanced and advanced polyps to cancer, with a wide spread of concentrations within each category. Fraser et al. [36] demonstrated a clear relationship between increasing faecal blood concentration, measured with the FOB Gold FIT, and pathological change in 375 fresh samples from participants of the Scottish programme. Ciatto et al. [20] used the FIT OC-Sensor and a population that included 191 cancers and 890 adenomas detected at colonoscopy to show increasing faecal haemoglobin concentration with increasing lesion severity and size. It remains 
a matter of conjecture whether all early-stage cancers bleed and whether they bleed intermittently, dependent perhaps upon on the mechanics of the gastrointestinal tract and the passage of digested foodstuffs. Intermittent or variable blood loss partially explains why the less-sensitive guaiac tests do not show consistently positive test results in patients who are later diagnosed with colorectal cancer and why, even with highly sensitive tests, 100 $\%$ clinical sensitivity is not achieved.

\subsubsection{Sample collection for faecal occult blood test devices}

Effective sample collection is critical to the success of a screening programme. The process of collection needs to be as simple as possible. Participants will always find the process inconvenient and unpleasant. Clear, simple and practical instructions are very important both to encourage participation and to the collection of a satisfactory specimen. The easier it is to present the stool for sampling and to transfer it to the test device, the greater the likely uptake to a screening programme. Current test kits use cardboard and wooden spatulas, plastic probes with serrated ends and brushes. Whilst most kits require the sample to remain away from the water in the toilet bowl before sampling, other devices sample the water that surrounds the stool. Many systems accept samples taken from toilet tissue paper. One RCT [23] showed that different sampling techniques can change FOBT screening compliance and two cross-sectional studies $[29,44]$ reported information on preference among different types of stool sampling methods. Practical experience has shown that in the age group commonly screened, physical and mental disabilities present a further reason for non-participation. Difficult sampling procedures with complex instructions greatly aggravate the inherent difficulties in collecting faecal samples.

Effective sampling is also important to the reliability of the test. Whilst the composition of faecal samples is affected by intestinal transit time, stool consistency [95], undigested foodstuffs and variable sample volume will also add to poor test performance. A technique that enables the sample to reflect blood throughout the stool is preferable and so a probe that can be inserted into various parts of the stool or a spatula or brush that enables collection of material across a large surface area must be better than single point sampling $[23,111,132]$. A well-designed RCT conducted in Australia on 1818 urban residents, aged 50-69 years, compared the participation rate of three screening cohorts [23]. The invited population used a wooden spatula (Hemoccult SENSA Beckman Coulter Inc. Fullerton, CA, USA), a spatula (FlexSure OBT Beckman Coulter Inc. Fullerton, CA, USA, three samples), and a brush (InSure Enterix Inc., Edison, New Jersey, USA, two samples) for sample collection. The overall participation rate was significantly higher in the InSure group (Hemoccult SENSA: 23.4\%, FlexSure: $30.5 \%$, InSure: $\left.39.6 \% \mathrm{X}^{2}=37.1, \mathrm{p}<0.00001\right)$. In a UK cross-sectional study [29] 1318 (50\%) of the eligible population $(n=2639)$ registered with two general practices were randomly selected and sent a three-page questionnaire to determine the acceptability of three methods of FOBT sampling, a sterile long stick transport swab, a conventional smear card with short wooden applicator and a scoop with collection pot. The swab was found most preferable and the smear-card the least preferred method of collection. A small cross-sectional study [44] compared toilet tissue and the short wooden applicator with the Hemoccult test but failed to show a statistical difference $(\mathrm{p}=0.05)$.

When applying a sample to the test device, consistent application of the required volume is important. Doubling the sample vol- ume can double the analytical sensitivity and halving it will halve analytical sensitivity. The thickness of the card surrounding the sample collection window on a guaiac test kit is important since it will influence the volume of sample transferred onto the window. A probe that, after collection, has to pass through a small hole to wipe off sample excess is an elegant system that is used in the HemSP, OC-Sensor and FOB Gold FIT, the latter two having devices that make use of a serrated probe. This collection method is used only for immunochemical devices and the probe surface, the number and depth of the groves in the serrated probe and the size of the hole through which the probe is inserted will affect the sample volume added to the buffer in the collection tube. Stool consistency will alter the volume of sample which adheres to the groves in the probe. Poor manufacturing tolerance will also contribute to a reduction in reproducibility of the sampling system.

\subsubsection{Guaiac faecal occult blood test - gFOBT}

The guaiac-based FOBT is still a commonly used method for detecting blood in faeces. The method exploits the pseudoperoxidase properties of the haem moiety in haemoglobin and liberates oxygen from 3-5\% dilutions of hydrogen peroxide in ethanol or methanol. The released nascent oxygen then reacts with alpha guaiaconic acid, the phenolic compound (2,5-di-(4-hydroxy-3methoxyphenyl)-3,4-dimethylfuran) present in guaiac, a resin extracted from a hardwood tree Guaiacum officinale (Lignum vitae). The reaction produces a compound with a quinine structure that rearranges by two-fold electron transfer to produce an unstable blue bis-methylene quinone dye.

Guaiac is still manufactured by extraction from tree resin and is therefore susceptible to batch variation. Batch variation is potentially a significant problem for population screening programmes for which a small change in analytical sensitivity could markedly change the referral rate for colonoscopy.

Guaiacum officinale is a tree native to South America and the Caribbean and is subject to Appendix II of the Convention on International Trade in Endangered Species (CITES) [60]. This is an international agreement regulating trade in endangered species in order to protect them from exploitation and extinction. Under CITES, export of specimens is subject to a government-issued permit certifying that they are legally obtained and that export will not be detrimental to the survival of the species.

In all current guaiac-based devices, the guaiac is absorbed into filter paper contained within a cardboard support. Faeces is applied by the participant to one side of the filter paper and, on receipt of the card, the laboratory applies an alcoholic solution of hydrogen peroxide to the other side of the paper. The volume of hydrogen peroxide added is not critical but the quantity of faeces applied is. The mass of the faecal sample will be influenced by the size of the application window and the thickness of the cardboard surrounding it. The hydrogen peroxide is usually applied from a dropper bottle and the laboratory staff look for the development of a blue colour within a time window prescribed by the kit manufacturer, typically 30-60 seconds. The blue dye is unstable and late reading will result in false negative results.

The test kit should have a means of checking performance; many kits will have a test positive and test negative quality control strip that develops alongside the participant's results and can highlight gross product or user errors. This QC strip should extend across the area used for clinical testing to enable identification of incomplete application of guaiac to the filter paper during product manufacture. 
Good kit design can greatly facilitate proper use. The identity of the card and participant should be easily and uniquely identified by the laboratory, usually by way of a barcode. Instructions and directions must be clear so that the sample is applied to the correct window. The design of the sample applicator needs to facilitate easy sample transfer and be suitable for the particular design of the kit. The size of the test window and the applicator must match to minimise marked under- or over-application of the sample. The device should carry the date the sample was applied so that the laboratory can disregard specimens that are too old to give reliable results.

Guaiac tests typically have an analytical sensitivity (limit of detection) of between 0.3 and $1 \mathrm{mg} \mathrm{Hb} / \mathrm{g}$ of faeces, but this will be affected by the sample loading levels and the time between collection and testing. The guaiac test can be made more sensitive $(0.15 \mathrm{mg} \mathrm{Hb} / \mathrm{g}$ ) by hydrating the sample on the test kit before adding hydrogen peroxide; that is the principal use in the Hemoccult Sensa, Beckman Coulter Inc. Fullerton, CA, USA.

\subsubsection{Immunochemical tests - FIT}

Unlike gFOBT, the utility of faecal immunochemical tests for haemoglobin (FIT) has been demonstrated only in one randomised controlled trial [121]; however the analytical superiority of immunochemical tests mean that they have recently become the test of choice for colorectal cancer screening programmes. FIT have been used for population screening in Japan since 1992 [103], and the OC-Sensor was approved for use in the U.S. by the Food and Drugs Administration (FDA) in 2001. Immunochemical tests can use monoclonal or polyclonal antibodies raised against human globin, the protein component of haemoglobin. The antibodies are attached to a latex particle, dye or an enzyme that in the presence of human globin forms a complex that can be detected by turbidity, aggregation (latex agglutination, haemagglutination and colloidal gold agglutination) or coloured dye produced by an enzyme. Since the protein structure of human globin is unique to humans, the immunochemical test should not be subject to interference from animal blood in the diet. Unlike haem, proteolytic enzymes gradually degrade globin as it moves through the intestine, and this confers on it more specificity for pathology in the distal intestinal tract than does haem. A variation of the immunochemical test marketed by Chemicon Europe Ltd, MonoHaem, uses antibodies against human globin to specifically immobilise haemoglobin and then the guaiac reaction to detect the haem.

FIT are typically 10-fold more expensive than gFOBTs [35]. Increased FIT test kit cost can be offset by the use of automated analysers and thus reduced staff costs and, where multiple gFOBT test cards are in use, by using a single FIT because adequate clinical sensitivity and specificity can be obtained using a single FIT. Immunochemical tests confer increased analytical specificity for human haemoglobin, and by using sensitive detection systems, they increase test sensitivity to low blood concentrations. FIT typically have limits of detection of less than $200 \mu \mathrm{g} \mathrm{Hb} / \mathrm{g}$ stool and can detect as little as $0.3 \mathrm{~mL}$ of blood added to a stool sample [102].

Faecal immunochemical tests for haemoglobin provide opportunities for improved population screening. HemSp, OC-Sensor and FOB Gold all use spectrophotometric measurement systems, sometimes with charged coupled devices (CCD), to measure the degree of agglutination, turbidity or the colour generated by the test. Automating instrument measurement increases test throughput and measurement precision, and eliminates user bias [36]. Instrumentation also provides an opportunity to manually adjust the cut-off limit below which the test is reported as negative and not referred for prospective colonoscopy.

Whilst the measurements performed on the buffered faecal sample using automated analysers can be quantitative, the impossibility of providing a reproducible sample means that these systems must not be considered capable of providing reliable quantitative test results in faeces. The gFOBT and FIT might both be considered semi-quantitative, the immunochemical test is analytically superior.

\subsubsection{Other tests}

o-Toluidine and benzidine have both been used as alternatives to guaiac but have been discontinued because they have been shown to be to be carcinogenic [52]. Imipramine and desipramine have also been described as alternative reagents to guaiac and have reports of less interference from vegetable peroxidases, iron and vitamin C, but they have not gained a place in the market [116]. Alpha guaiaconic acid, the active component of guaiac gum, has been synthesised but proved unstable and unsuitable as an alternative to the tree extract, which may contain contaminants with stabilising properties.

The measurement of porphyrins produced by the action of intestinal bacteria on haemoglobin provides an alternative method for measuring blood in faeces $[2,3,105]$ and recently mass spectrophotometric methods have been described, but they are unlikely to be adopted for population screening.

The literature describes many alternative biomarkers for the presence of colorectal cancer. These markers includes albumin, haptoglobin, transferrin, pyruvate kinase (PK) isoenzyme type M2, calprotectin, Ca3 anaphylatoxin, colon-specific antigen (CCSA-3 and CCSA-4) and a variety of DNA-related markers.

When used alongside guaiac-based or immunochemical devices, PK isoenzyme type M2 has not shown adequate specificity for population screening $[83,108]$. Calprotectin has a role in identifying patients with inflammatory bowel disease, but a metaanalysis of the literature in 2006 concluded that it was unsuitable for screening for colorectal cancer [125].

The use of molecular biology techniques to identify cancer-related DNA or protein biomarkers, used singly or as a panel, shows promise but is in its infancy. The use of DNA microarrays to detect the present of mutations in genes such as TP53, K-ras, APC, BAT26 and BRAF might bring us closer to earlier detection. A study of 5486 asymptomatic patients by Imperiale in 2004 showed increased sensitivity and specificity for invasive cancer and advanced neoplasia using faecal DNA relative to gFOBT, but failed to detect over $50 \%$ in each group [56]. A recent paper by Wang $\&$ Tang [126] showed the hypermethylated SFRP2 gene in faecal DNA to be a candidate colorectal biomarker, but none of these DNA-related markers has been demonstrated to have the necessary characteristics to qualify them for use in population screening. In Young's review of new screening tests he remarks that the epigenetic marker for the methylated vimentin gene has improved sensitivity for cancer but that its overall performance relative to existing gFOBT and FIT remains unclear $[18,130]$. In a 2008 review of the cost-effectiveness of faecal DNA, immunochemical and guaiac-based tests using the Markov model, the authors conclude that blood markers remain the preferred option in high-adherence populations [88]. A Medline review of new stoolbased tests by Haug concluded that "while promising performance characteristics have been reported for some tests, more persuasive evidence from larger, prospectively designed studies... 
was needed" [49]. Currently the new markers are both expensive and show very poor sensitivity to cancer and adenomas.

In the short term, marker tests based on gene or epigenetic mutations may show merit for use in screening selected high-risk populations or for monitoring disease progression or recurrence, but in the long term we may see them as the preferred markers for general population screening.

\subsubsection{Recommendations}

\section{Guaiac faecal occult blood tests}

Guaiac faecal occult blood tests have proven characteristics that make them suitable for population screening. They lack the analytical specificity and sensitivity of immunochemical tests, their analysis cannot be automated and the Hb concentration at which they turn from negative to positive cannot be adjusted by the user. For these reasons guaiac-based tests are not the preferred test for a modern population screening programme, although depending on local labour costs, the mechanism of kit distribution and collection, and reduced sample stability in immunochemical testing, they might prove more practicable and affordable than immunochemical testing (Sect. 4.2.4, 4.3 and 4.4.2) (I-B). ${ }^{\text {Rec } 4.1}$

\section{Faecal immunochemical tests for haemoglobin}

Immunochemical tests have improved test characteristics compared with conventional guaiac-based tests. They are analytically and clinically more sensitive and specific, their measurement can be automated and the user can adjust the concentration at which a positive result is reported. Immunochemical tests are currently the test of choice for population screening; however, individual device characteristics including ease of use by the participant and laboratory, suitability for transport, sampling reproducibility and sample stability are all important when selecting the FIT most appropriate for an individual screening programme (Sect. 4.2.5, 4.3 and 4.4.2) (II-A). ${ }^{\operatorname{Rec} 4.2}$

\section{DNA and other related new markers}

Only tests for blood in faeces have been demonstrated to have the necessary characteristics to be suitable for population screening. DNA and other related new markers are currently unsuitable for screening, either singly or as members of a panel of tests (Sect. 4.2.6) (III-D). ${ }^{\operatorname{Rec} 4.3}$

\subsection{Analytical characteristics and performance $\nabla$}

\subsubsection{Analytical sensitivity}

Analytical sensitivity or limit of detection describes the lowest concentration that an analytical system can detect with confidence. The detection system used by FIT makes the test inherently more sensitive than guaiac-based systems. The concentration units quoted for analytical sensitivity depend on the method used for determination, for example $\mathrm{mL}$ of blood/g or $\mathrm{mL}$ of faeces, or mg (or $\mu \mathrm{g}$ ) of haemoglobin/g or $\mathrm{mL}$ of faeces. Most manufacturers and scientific papers quote $\mathrm{mg} \mathrm{Hb} / \mathrm{g}$ faeces. The haemoglobin content should be determined with knowledge of the haemoglobin concentration in the blood used, and faeces should be measured as the wet weight of a formed stool sample. Some manufacturers and studies also quote the concentration of haemoglobin not in faeces but in the buffer solution used for analysis, and this is different for different devices, making simple comparison of device sensitivity difficult e.g. the HemSp devices carry $0.3 \mathrm{mg}$ faeces $/ \mathrm{mL}$ buffer and OC-Sensor $10 \mathrm{mg}$ faeces $/ \mathrm{mL}$ buffer.
Given the variable consistency of faecal samples and the dependence upon diet and intestinal transit time, the relationship between patient samples and test samples prepared in the laboratory is often a poor one. Manufacturers may quote sensitivity on blood solutions rather than spiked faecal samples and if quoted for faecal samples, the time period between in-vitro addition of blood to faeces and analysis is unlikely to be typical of that between participant sampling and analysis in a screening programme. The unstable nature of samples used in FOBTs is discussed later in this chapter.

\subsubsection{Analytical sensitivity and cut-off limits}

Until recently it has not been possible to adjust the analytical sensitivity of FOBTs and so adjust the proportion of positive tests. This facility to adjust sensitivity is still not available for gFOBTs, with the exception of the simple process of hydrating the specimen before testing. With Hemoccult SENSA this process increases test sensitivity but at the expense of specificity, thereby increasing the false-positive rate [77, 93].

Point-of-care FIT typically use an immunochromatographic technique that produces a coloured line where the antibodies and haemoglobin are immobilised. The presence of the line is detected by eye, and the limit of detection is dependent upon the configuration of the device, the characteristics of the antibodies and chromogens and the visual acuity of the reader. These FIT devices are suitable for small-volume point-of-care testing but are unsuitable for population screening and do not provide numeric results.

The heterogeneous nature of faeces and the inherent inconsistency in sample collection makes reliable quantitative measurement of blood in faeces impracticable. However, many of the automated immunochemical test devices that are suitable for population screening provide a numeric analytical result for the sample presented for analysis. These systems determine the turbidity or colour density of a reaction between haemoglobin and the antibody/chromogen system. Measurement is usually performed in a cuvette containing an aliquot of sample in buffer and added reagents (OC-Sensor, FOB Gold).

Whilst the results provided by these systems must not be considered quantitative measures of faecal haemoglobin, the numeric results provide an opportunity to select a cut-off limit above which a test can be defined as positive. This feature enables the user to adjust the positivity rate and thereby the clinical sensitivity and specificity of the test. Such a system enables colonoscopy referral rates to meet the available colonoscopy resource. The clinical implications of manipulating the cut-off limit and/or the number of samples used for analysis is described later in this chapter.

- Table 4.1 gives the analytical sensitivities quoted by manufacturers for a range of FOBT devices. Differences in quoted analytical sensitivity may reflect the use of different methods of assessment as well as product characteristics.

\subsubsection{Analytical specificity and interference}

In the context of gFOBT and FIT, analytical specificity is the ability of the test to detect human blood accurately without interference from other endogenous or exogenous components of the faeces. It does not include interference from blood produced from pathological or physiological sources, which is termed biological interference since the interference is not as a result of a weakness in the analytical system. 
Table 4.1 Analytical sensitivities.

\begin{tabular}{|lll}
\hline Product name & Manufacturer/Supplier & Analytical Sensitivity \\
\hline Guaiac-based test & & $0.9 \mathrm{mg} \mathrm{Hb} / \mathrm{g}$ \\
\hline Coloscreen & Helena Laboratories, Texas, USA & $0.6 \mathrm{mg} \mathrm{Hb} / \mathrm{g}$ \\
\hline Hema-Screen & Immunostics Inc. 3505 Sunset Avenue, Ocean, New Jersey, 07712, USA & $30 \%$ positivity at 0.3 mg Hb/g \\
\hline Hemoccult & Beckman Coulter Inc. Fullerton, CA 92835, USA & $75 \%$ positivity at 0.3 mg Hb/g \\
\hline Hemoccult SENSA & Beckman Coulter Inc. Fullerton, CA 92835, USA & $1.05 \mathrm{mg} \mathrm{Hb} / \mathrm{g}$ \\
\hline MonoHaem & Chemicon Europe Ltd & $6 \mathrm{mg} \mathrm{Hb} / \mathrm{g}$ \\
\hline Hema-Check & Siemens PLC & $2 \mathrm{mg} \mathrm{Hb} / \mathrm{g}$ \\
\hline HemaWipe & Medtek Diagnostics LLC, supplier; BioGnosis Ltd & $40 \mathrm{\mu g} \mathrm{Hb} / \mathrm{g}$ \\
\hline Automated Immunochemical Test/Analyser & & $10 \mathrm{ng} \mathrm{Hb} / \mathrm{mL}$ \\
\hline OC-Sensor/OC-Sensor Diana \& OC-Sensor Micro & Eiken Chemical Co., Tokyo, Japan & $14 \mathrm{ng} \mathrm{Hb} / \mathrm{mL}$ \\
\hline HemSp/MagStream HT & Fujirebio Inc. Japan & Medinostics Products \\
\hline FOB Gold/SENTiFOB analyser & Supplier; Sentinel Diagnostics SpA, Milan, Italy & \\
\hline
\end{tabular}

\subsubsection{Analytical interference}

gFOBTs use a non-specific reaction for detecting blood and whilst cheap and simple to use, they are inherently susceptible to positive interference from oxidising agents and compounds with oxidase or peroxidase properties. gFOBTs are also subject to negative interference from compounds with reducing properties such as vitamin C. In its 2007 guidance to industry, the US FDA Center for Devices and Radiological Health illustrated the range of dietary substances known to interfere with gFOBTs: broccoli, cantaloupe, cauliflower, horseradish, parsnip, red radish, turnip, iron and vitamin C supplements, and haemoglobin from beef, chicken, fish, horse, goat, pig, rabbit and sheep.

Evidence suggests that although the gFOBT test is open to interference from normal diets, this is not substantial and is reported to be negated by a time delay of at least $48 \mathrm{~h}$ between sample collection and analysis [110]. A diet including $750 \mathrm{~g}$ of raw peroxidase-rich fruit and vegetables daily is reported to cause false positive results, although $750 \mathrm{~g}$ is an unusually large daily consumption. A systematic review of the effect of diet on gFOBT showed that dietary restriction was not necessary [90]. The five randomised trials included in the review all used gFOBT Hemoccult tests. None of the studies showed a statistically significant difference between the group in which peroxidise-containing food (red meat, no red meat, poultry, fish, or certain raw vegetables and fruits), nonsteroidal anti-inflammatory drugs (NSAIDs, including aspirin), and vitamin $\mathrm{C}$ were prohibited compared with a control group without dietary restrictions (meta-analysis: absolute difference in positivity rate $0 \% ; 95 \% \mathrm{CI},-1 \%$ to $1 \%$ ). A cohort study conducted in Israel by Rosen, Knaani \& Samuel [97] on 944 asymptomatic subjects attending colorectal cancer screening (mean age 60.2 \pm 11.1 ) reported an overall gFOBT positive rate of $7.5 \%$, while neoplasia was found in $16(22.5 \%)$ subjects with positive gFOBT. Among subjects with and without dietary restriction, the positivity rates were $7.2 \%$ and $5.5 \%$ respectively $(\mathrm{p}=$ 0.26 ). These positivity rates are markedly higher than those observed in the UK screening pilots (1.6\% in England and $2.1 \%$ in Scotland with an average of $1.9 \%$ ) and are now observed in the fully rolled-out screening programme which does not advocate dietary restriction [117].

FIT brings a significant improvement in analytical specificity. The use of a specific antibody against human globin makes cross-reactivity with dietary haemoglobin very unlikely, and the method used for detecting the antibody reaction can also be made largely free from interference from other dietary interference. Studies have not been published that demonstrate whether the reagents used in FIT will detect haemoglobin variants. Polyclonal assays are unlikely to show cross-reactivity problems, but manufacturers should provide evidence that their analytical systems react similarly with all known haemoglobin variants. A recent evaluation has shown that with $\mathrm{HbA1c}, \mathrm{HbS}, \mathrm{HbC}, \mathrm{HbD}, \mathrm{HbE}$ and $\mathrm{HbF}$ using the HemSp/MagStream HT, OC-Sensor Diana and FOB Gold Sentinel Systems, only HbF showed poor recovery and might give false negative results [64].

Instant-View is an FIT that was used by the Australian health service, and since it requires sampling from the toilet bowl it is subject to other potential analytical interferences. In their US FDA 510(k) submission, the US supplier of Instant-View, Alfa Scientific Designs, disclosed decreased analytical sensitivity in the presence of toilet bowl deodorizers, fresheners and cleaner, and required that toilet bowl deodorizers, fresheners or cleaners be removed from the toilet bowl before collecting samples and that the toilet be freshly flushed.

- Table4.2 lists known gFOBT interferences. A good account is included in the MHRA Report of 2000 and summarized by Starkey [113].

\subsubsection{Biological interference}

Any physiological process or non-colorectal cancer-related pathological lesion that increases the loss of blood into the intestine is a source of biological interference. Although aspirin and NSAIDs pose potential interference, studies have shown either no effect or an increased sensitivity to the detection of cancer and adenomas among those who are taking aspirin or NSAIDs.

\section{Aspirin and NSAIDs}

One double-blind RCT and one cohort study investigated whether the use of regular aspirin or NSAIDs is a risk factor for a falsepositive FOBT result. A double-blind RCT [43] was conducted on 40 healthy volunteers aged $29.8 \pm 0.6$ years who were randomised to placebo, or doses of $30 \mathrm{mg}, 81 \mathrm{mg}$, or $325 \mathrm{mg}$ of aspirin. Short-term (30 days) use of low-dose aspirin did not induce sufficient intestinal injury to cause positive FOBTs (number of GI erosions aspirin group: $6 / 30(20 \%)$; placebo: $1 / 10(10 \%) p=$ $0.66)$. A cohort study [59] showed no difference in the prevalence of colonoscopic findings that would potentially explain a positive FOBT result between regular aspirin or NSAID users and nonusers, even after adjusting for factors that affect the risk of a lesion that would account for a positive result (absolute difference $2 \%(95 \% \mathrm{CI}, 10-14), \mathrm{p}=0.7)$. The study also found no relationship between the dose of aspirin and the likelihood of colonoscopic 


\begin{tabular}{|c|c|c|}
\hline Positive interference & Comment & Reference \\
\hline $\begin{array}{l}\text { Non-human blood (beef, pork, chicken, pheasant, } \\
\text { salmon, sardines, black pudding, German blutwurst, } \\
\text { French boudin noir, Spanish morcilla and liver) }\end{array}$ & $\begin{array}{l}\text { Reduced by cooking. } \\
\text { Avoid red meat for } 3 \text { days prior to sampling. } \\
\text { Meta-analysis suggests dietary restriction not necessary }\end{array}$ & {$[34,53]$} \\
\hline Myoglobin & & {$[1,6,74,106,127]$} \\
\hline Iron & Mixed reports about whether iron supplements interfere & \\
\hline Providone-iodine antiseptic & $\begin{array}{l}\text { Use on perianal area or in urinary catheters should be avoided since iodine } \\
\text { will oxidise guaiaconic acid }\end{array}$ & [101] \\
\hline Contact with toilet sanitizers in toilet water & $\begin{array}{l}\text { Potential for negative and positive interference. gFOBT less than FIT. } \\
\text { Reported in chlorine-releasing agents }\end{array}$ & [54] \\
\hline $\begin{array}{l}\text { Raw fruits \& turnips, broccoli, horseradish, cauli- } \\
\text { flower, cantaloupe, parsnip and red radish }\end{array}$ & $\begin{array}{l}\text { Large daily consumption only causes interference. Caused by peroxidases } \\
\text { that act like haemoglobin and give false positives. } \\
\text { Cooking for } 20 \text { mins at } 100^{\circ} \mathrm{C} \text { destroys peroxidases and a delay of } 2 \text { days } \\
\text { between collection and analysis is also effective as long as a non-hydrated } \\
\text { gFOBT is used }\end{array}$ & {$[53,110]$} \\
\hline Negative interference & Comment & Reference \\
\hline Vitamin C (Ascorbic acid) & $\begin{array}{l}\text { Reducing agents counters oxidising effect on guaiaconic acid. Vitamin C } \\
\text { intake should be }<250 \mathrm{~m} / \mathrm{d} \text {. Normal diet unlikely to interfere but high dose } \\
\text { supplements might do so }\end{array}$ & {$[39,57,58]$} \\
\hline Degradation of haem & $\begin{array}{l}\text { Haem degrades slowly, a process that is accelerated if the faecal sample } \\
\text { remains moist and warm }\end{array}$ & $\begin{array}{l}\text { CEP Report } 2006 \\
{[11]}\end{array}$ \\
\hline Contact with toilet sanitizers in toilet water & Potential for negative and positive interference. gFOBT less than FIT & [54] \\
\hline
\end{tabular}

findings (chi-squared test for trend $\mathrm{p}=0.6$ ). Overall, advice to patients to restrict their diet and avoid NSAIDs and vitamin C does not appear to change positivity rates. This finding was consistent across all studies, regardless of the intensity of the restriction. A recent report by Levi et al. [69] showed an increase in sensitivity but no loss of specificity of FIT (OC-Sensor) for detection of cancer and advanced adenomas in those using aspirin/NSAIDs or anticoagulants.

\section{Anticoagulants}

Anticoagulants present a further source of biological interference. The effect of anticoagulants on the false-positive rate in a population-based FOBT screening programme was evaluated in two studies [12, 21]. The cohort study conducted within the Scottish arm of the national colorectal cancer screening pilot on 846 subjects aged 50-69 years old showed that taking anticoagulant medication (aspirin, COX-2 inhibitors, other NSAIDs and warfar- in) at the time of testing is associated with a statistically significant $6.4 \%$ increased rate of negative colonoscopy. Diagnosis of colorectal neoplasia was higher in the no-anticoagulant group compared with the anticoagulant medication cohort (56.5\% vs $47.5 \%$; absolute difference $9 \%, p=0.012$ ). A study in an American healthcare system programme looked at all patients taking warfarin who were referred for the evaluation of a positive FOBT [12]. For each patient taking warfarin, an age- and gender-matched control was enrolled. The positive predictive value (PPV) of FOBT for gastrointestinal lesions consistent with occult blood loss in patients taking warfarin was similar to that in the ageand gender-matched control group of subjects with a positive FOBT who were not taking oral anticoagulants $(59.0 \%, 95 \% \mathrm{CI}$, $52.3-65.8 \%$; 53.8\%, 95\% CI, 47.0-60.6\%; $\mathrm{p}=0.27$ ).

- Table4.3 summarises sources and mechanisms of biological interference which will reduce the specificity of either gFOBT or FIT analysis.

Table 4.3 Biological interferences.

\begin{tabular}{|c|c|c|}
\hline Physiological & Comment & Reference \\
\hline \multicolumn{3}{|l|}{ Loss from the gums after vigorous teeth brushing } \\
\hline Menstrual bleeding & - & - \\
\hline \multicolumn{3}{|l|}{ Pathological } \\
\hline Inflammatory bowel disease (Crohn's disease, colitis) & & [94] \\
\hline Gastritis from alcohol or chemotherapeutic drugs & - & \\
\hline Gastric cancer & & {$[135,136]$} \\
\hline Anti-inflammatory drugs & Increased blood loss of $1-2 \mathrm{~mL} / \mathrm{d} .5 \%$ of those on high dose NSAIDs lost & {$[80]$} \\
\hline (ibuprofen, naproxen, corticosteroids, phenylbutazone) & $5 \mathrm{~mL} / \mathrm{d}$ & [69] \\
\hline Aspirin & $\begin{array}{l}\text { No FIT interference reported in low dose aspirin. High-dose blood loss } \\
5 \mathrm{~mL} / \mathrm{d}\end{array}$ & $\begin{array}{l}{[3,80]} \\
{[69]}\end{array}$ \\
\hline $\begin{array}{l}\text { Proximal intestinal tract inflammation (gastritis, oesophagitis } \\
\text { and gastric and duodenal ulceration) }\end{array}$ & & [94] \\
\hline Anticoagulation therapy & 2005 study showed no effect from warfarin & [12] \\
\hline Perianal bleeding & - & - \\
\hline
\end{tabular}




\subsubsection{Dietary and drug restrictions}

Potential interference of diet and drug intake on test performance has been pointed out above (Sect. 4.3.2.1 and 4.3.2.2) and the organisational aspects of drug and dietary restriction are discussed in Ch. 2 [76] (Section 2.5.1.2). Whilst most gFOBT manufacturers recommend dietary advice, the potential detrimental impact on participation rates makes it unattractive. One study used an immunochemical test and compared the participation rates of two groups, one with and one without dietary restriction [22]. Two further studies [23,31] compared participation rate in a guaiac test with dietary restriction and in an immunochemical test without dietary restriction. Predictably, all three studies found greater participation when the diet was unrestricted. However, these studies and their data are not sufficient to exclude the possibility of other factors contributing to the outcomes.

\subsubsection{Other factors influencing analytical performance}

\subsubsection{Prozone effect}

Immunochemical analysis is prone to giving falsely low results when the analyte being tested is at markedly elevated concentrations. This well-described interference is called the prozone or "hook" effect. The concentration of haemoglobin at which an FIT exhibits this effect needs to be very high and should be disclosed by the manufacturer. If an analytical method exhibits a prozone effect, then the measurement system should be able to detect erroneous results and warn the analyst. This is a requirement of $\mathrm{U}$. S. FDA 510(k) submissions.

\subsubsection{Sample quality}

The quality of the sample is very important; it must be reproducible and representative of the stool, of the required volume and be adequately preserved. Many of the issues that impinge on sample quality have been discussed earlier. The stability of haemoglobin in faeces is an important consideration when selecting the preferred test, developing arrangements for sample transport to the laboratory and determining the urgency of analysis on the arrival of samples in the laboratory.

The haem moiety used in gFOBTs is more stable than the globin moiety used in FIT. Transport of a dried sample, which is used for most guaiac test kits, provides greater stability than that in wet buffer which is usually used for immunochemical tests. The acceptable time period between sampling and testing is defined by the product manufacturers in their instructions for use. For gFOBTs the maximum time period is usually between 14 and 21 days; for FIT it is much less.

Haem in haemoglobin is degraded slowly after collection; if samples are collected onto filter paper, the design of the test device and envelope should maximise the speed of drying and so help preserve the sample. Young et al. demonstrated the deterioration of wet samples in a study using gFOBT in 1996 [131]. The UK NHS MHRA report of 2000 illustrated the influence of excessive sample loading, high temperature storage, and exposure to sunlight on 12 occult blood kits [89]. The UK NHS CEP report of 2006 reported the effect of sample storage time upon positivity for four gFOBT kits, the change from positive to negative test result being most marked with those test kits that have the lowest limit of detection [11]. For gFOBT, a regression study by Faure et al. investigated the influence of temperature and moisture on gFOBT sensitivity. In this study it was observed that the positivity rate of Hemoccult II in a 10-year screening programme showed a signifi- cant change between $1.61 \%$ in summer to $2.80 \%$ during the winter [30]. No significant effect of temperature alone was observed: the positive rate decreased from $74.0 \%$ at $4{ }^{\circ} \mathrm{C}$ in the presence of silica gel to $68.0 \%$ at $30^{\circ} \mathrm{C}$ in the presence of water $(p=0.52)$. In this study the decrease in positive rate due to the presence of moisture was statistically significant $\left(84.0 \%\right.$ at $4{ }^{\circ} \mathrm{C}$ and $100 \%$ humidity, $58.0 \%$ at $25^{\circ} \mathrm{C}$ with silica gel; $\mathrm{p}=0.007$ ).

Globin in haemoglobin is an easily degraded protein moiety and more susceptible to degradation than haem. Proteolysis of globin should be minimised between sample collection and analysis. Whilst appropriate constituents in collection buffer solutions might reduce degradation, the stability of globin in the wet collection systems used by most FIT is poor compared with haem used in gFOBTs. The concentration of haemoglobin in the buffer solutions after sampling can be very low, typically $20 \mathrm{ng} / \mathrm{mL}$ with the collection device used by the MagStream HT. At these low concentrations the haemoglobin molecule is susceptible to decomposition and may be adsorbed onto the surface of the collection vessel and measurement cuvette. Buffers that are rich in proteins such as bovine serum albumin (BSA) and haptoglobin can minimise adsorption and help stabilise the haemoglobin. Unpublished data from the manufacturers of the immunochemical devices HemSp and OC-Sensor show good stability at refrigerator temperatures $\left(4^{\circ} \mathrm{C}\right)$ but marked deterioration with rising temperature. Vilkin et al. [122] and Rosen et al. [99] showed, over 21 days, no significant change at $4^{\circ} \mathrm{C}$ or $20^{\circ} \mathrm{C}$ but a daily fall of $3.7 \% \pm 1.8$ at $28^{\circ} \mathrm{C}$ with the FIT OC-Micro system (Eiken Chemical Co., Tokyo, Japan). Rozen used storage in a refrigerator and supplied an opaque double zip-lock bag for such storage. Fraser et al. [37] reported the successful use of dried samples for FIT using two Immunostics products (Immunostics Inc. Sunset Avenue, Ocean, New Jersey, USA). Hema-Screen Devel-ATab was used to collect the sample and Hema-Screen SPECIFIC as the immunochemical assay system. The low concentrations of haemoglobin detectable in FIT devices increases susceptibility to stability problems. Whilst sample stability has not presented a major difficulty for programmes using gFOBTs, it is likely to do so for those adopting wet sample collection with FIT. The acceptable time between collection and analysis is markedly influenced by ambient temperature during storage and transport, and this will depend on transport and weather conditions.

Between December 2008 and May 2009, the Australian Screening Programme encountered stability problems with the HaemSp/ MagStream HT system [9]. Positivity levels fell markedly during the 6-month period, and participants required retesting. Very high summer temperatures and the introduction of a new buffer with a lower protein concentration may have contributed to haemoglobin instability in this programme and a consequent reduction in positivity rates. A recent report describes retrospective analysis of measured haemoglobin over several years by the screening programme in Northern Italy [40]. The study reveals significant seasonal variation in the positivity rates of the OCSensor FIT that may be attributed to high summer temperatures. Manufacturers of FIT devices specify stringent storage and transit conditions to minimise the sample deterioration. These conditions present a practical challenge to the organisation of FITbased screening programmes. 


\subsubsection{Device consistency}

The ability of FIT and gFOBT kits to maintain consistent performance across reagent batch changes and product redesigns is important for population screening since minor changes in product sensitivity and specificity can greatly change the number of patients referred for colonoscopy. Companies need to be able to demonstrate good quality manufacturing practice and quality assurance procedures that minimise batch-to-batch variation. Guaiac gum is a natural product and is therefore more susceptible to product inconsistency than manufactured monoclonal antibody reagents that can be used by FIT. Polyclonal antibodies, which are used for each of the current automated FIT, are susceptible to batch-to-batch variation, and therefore an understanding of the batch size of all reagent components is important. In a market with many small manufacturers, the long-term viability of the product and company should also be considered.

\subsubsection{Analytical quality assurance - internal quality control (IQC) and external quality assessment schemes (EQAS)}

Rigorous analytical quality assurance procedures must be adopted by laboratories providing gFOBT and FIT analysis for population screening. To minimise analytical and procedural variability, the number of laboratories used for population screening should be small. In the English programme, laboratories typically serve a population of $10-15$ million, approximately $10-16 \%$ of which will be within the screening age group. All laboratories providing screening services should be associated with a laboratory accredited to ISO 15189:2007, Medical laboratories-Particular requirements for quality and competence (http://www.iso.org/iso/ iso_catalogue.htm). The laboratory should be led by a qualified clinical chemist who is trained and experienced in the techniques used for analysis and in clinical quality assurance procedures. The laboratory staff should be appropriately trained and competent in the use of the analytical device/instrumentation, quality control and assessment procedures and associated information technology.

For those laboratories using visually read gFOBTs, the design of the test kit will influence the reliability of analysis. Reproducibility in detecting the blue gFOBT colour in the presence of dark faecal pigments depends on good staff training and experience but can be improved by other factors. The visual acuity and colour perception of the reader should be checked professionally and monitored. The colour of the test card surrounding the sample, the colour of surrounding walls and the colour, temperature and brightness of artificial lighting all should be considered. The opportunity for errors due to operator fatigue should be minimised by enforcing periodic work breaks. The competence of staff to perform visual tests should be checked before they commence each batch of analysis, typically using pre-loaded test kits with known positivity that is hidden from the operator. A rigorous monitoring system should be adopted to identify staff who have spot positivity rates that are markedly different from the mean or who exhibit marked variability.

Most gFOBTs and point-of-care FIT devices have a means of checking the integrity of the device and reagents by way of a quality control check integral to the device. For gFOBT, this control can check whether guaiac has been applied across the whole of the test area and whether the hydrogen peroxide reagents are working correctly. Point-of-care FIT devices provide a similar check of reagent integrity but are unsuitable for population screening.
The case for automation in population screening programmes is a strong one, and should significantly influence the choice of an acceptable occult blood testing system. Automated FIT analysis will require internal quality control procedures appropriate to the chosen technique and instrument. As a minimum, laboratories should adopt the manufacturers' instructions for use, and give consideration to what additional internal quality control measures can be used to check instrument accuracy and imprecision throughout the period of analysis. Good analytical performance is particularly important at the selected cut-off concentration, and quality control measures should reflect that requirement. Participation in an external quality assessment scheme (EQAS) is seen as mandatory for tests performed in a clinical laboratory. Participation in an EQAS enables assessment of bias between participating laboratories, and is particularly important for a national screening programme utilising several laboratories. The availability of an EU-wide EQAS is desirable. National population screening programmes should have quality assurance procedures that enable oversight of the analytical performance of all screening laboratories. Satisfactory performance in an EQAS provides an objective criterion of competence.

A summary of the three FIT systems that have some of the characteristics suitable for population screening is provided in $\square$ Table 4.4.

\subsubsection{Recommendations}

\section{Sample stability between collection and analysis}

Whilst a maximum period of 14 days between collection and analysis is frequently quoted for many guaiac faecal occult blood tests, that quoted for immunochemical tests is significantly shorter. Until more stability data are published, screening programmes should adopt the conditions and period of storage described in manufacturer's instructions for use having determined that they are appropriate for local conditions that might expose samples to high temperatures for long periods of time (Sect. 4.3.3.2) (III-A). ${ }^{\text {Rec } 4.4}$

\section{Test interference - drug and diet restriction}

Dietary constituents present potential interference in guaiac faecal occult blood tests. Dietary restriction has not been demonstrated to significantly increase screening specificity, and risks reducing participation rate. The potential for dietary interference is significantly less for immunochemical tests. With the qualification that a diet peculiar to a particular country or culture may not have been tested or reported dietary restriction is not indicated for programmes using either guaiac-based or immunochemical tests (Sect. 4.3.2.1, 4.3.2.3) (II-D). ${ }^{\text {Rec } 4.7}$

Interference from bleeding associated with drugs such as aspirin, nonsteroidal anti-inflammatory drugs and anticoagulants (e.g. warfarin) present potential interference in both guaiac and faecal immunochemical tests for haemoglobin. Although the literature carries some contradictory reports of the effect of anticoagulants on screening outcome, drug restriction is not recommended for population screening programmes using either guaiac-based or immunochemical tests (Sect. 4.3.2.2, 4.3.2.3) (III-D). . Rec 4.8 $^{\text {. }}$ 
Table 4.4 Comparative table of automated FIT.

\section{HemSP/MagStream HT}

Alternative name(s): Developed from Immudia-HemSp

(Marketed as HaemSelect in the US)

Manufactured by: Fujirebio Inc. Japan

Sold by: Fujirebio Europe B.V.

(http://www.fujirebio.co.jp/english/index.html)

Principle of measurement system: MagStream HemSp ${ }^{\circledR}$ is based on magnetic particle agglutination. The faecal specimens are incubated with magnetic gelatine particles which are ferrite and gum arabic coated with rabbit anti-human haemoglobin antibodies. The solid particles are collected in the centre of microplate wells by magnetic attraction and inclined to about 60 degrees and examined for change in particle aggregates. In the presence of human haemoglobin, the particles remain aggregated in a spot with minimal change (positive result). In the absence of human haemoglobin, particles flow down the slope (negative result). The appearance of particle aggregates is interpreted by MagStream HT using CCD image capture and computer determination of the length of the line of magnetic particles. The company recommends that 1 of 2 samples needs to be positive and state that the measurement system has not been designed for quantitative measurement. This system has been developed to give a sharp cut-off at a concentration of $20 \mathrm{ng} / \mathrm{mL}$ and not to provide quantitative measurements for user-defined cut-off concentrations, and is not CE marked for this purpose.

Recommended number of separate samples used for assessment: 2 samples

Method of sample collection: Stick in buffer held within the device Means of reading: MagStream HT, an automated instrument that holds 400 samples and has a memory capacity of 2 million test results Speed of analysis: 960 tests per hour (MagStream HT) Quantity collected by sampling device: $0.3 \mathrm{mg}$ of faeces Volume of buffer in collection device: $1 \mathrm{~mL}$

Analyser sample volume: $25 \mu \mathrm{L}$

Quality control: Standard laboratory QC procedures

Mailing acceptable to EU: It is being used in both France and Slovenia.

Cut-off level: Not designed or CE marked for an adjustable cut-off

Limit of detection: $10 \mathrm{ng} / \mathrm{mL}$

Use in population screening: Japan, France and Slovenia

Recent pertinent scientific papers: $[67,81,82]$

Website URL: Fujirebio

Fujirebio Inc Japan

http://www.fujirebio.co.jp/english/product/immunological.html
OC-Sensor

Alternative name(s): OC-Hemodia, OC light (not available in EU) Manufactured by: Eiken Chemical Co., Tokyo, Japan

Sold by: Mast (UK), Alfa Wassermann (Italy), Pharmatrade (Israel) Principle of system: Latex agglutination using polystyrene latex particles coated with polyclonal anti haemoglobin Ao antibodies. The assay uses a 6-point standard curve, and measurement is made at $600 \mathrm{~nm}$ with an algorithm that uses a kinetic endpoint.

Recommended number of separate samples used for assessment: 1 sample

Method of sample collection: Serrated stick in buffer held within the device

Means of reading: OC-Sensor Diana \& OC-Sensor Micro (successor to OCSensor $\mathrm{Neo}$ ) are both automated instruments and are both CE marked. The Diana has a memory capacity for 100000 test results

Speed of analysis: 280 samples per hour (OC-Sensor Diana)

Quantity collected by sampling device: $10 \mathrm{mg}$ of faeces

Volume of buffer in collection device: $2 \mathrm{~mL}$

Analyser sample volume: $35 \mu \mathrm{L}$

Quality control: Standard laboratory QC procedures

Mailing acceptable to EU: Reported to have been agreed by the UK post office

Cut-off level: CE marked for a user-defined cut-off. Default setting $100 \mathrm{ng} / \mathrm{mL}$

Limit of detection: $20 \mathrm{ng} / \mathrm{mL}$ in buffer

Use in population screening: The Netherlands [120, 121], Northern Italy [16], US, Uruguay [32] and France

Website URL: http://www.eiken.co.jp/en/company/index.html URL: http://www.eiken.co.jp/en/product/index.html\#anc03

\section{FOB Gold}

Manufactured by: Sentinel Diagnostics SpA, Milan, Italy Principle of system: The $F O B$ Gold reagents use an antigen-antibody agglutination reaction between human haemoglobin and polyclonal anti-human haemoglobin antibodies coated on polystyrene particles. Agglutination is measured as an increase in absorbance at $570 \mathrm{~nm}$ and is proportional to the concentration of human haemoglobin contained in the sample. The calibrator is a lyophilized material containing human haemoglobin, and this is used to generate a six-point calibration curve using serial dilutions of the reconstituted material. The manufacturer provides lyophilized quality control preparations at two haemoglobin concentrations. The total reading time is 8 minutes.

Means of reading: The FOB Gold reagents can be used on any suitable immunoassay automated analyser although the manufacturer provides the SENTIFOB analyser

Speed of analysis: 75 tests/hr (SentiFOB)

Quantity collected by sampling device: $10 \mathrm{mg}$ of faeces

Volume of buffer in collection device: $1.7 \mathrm{~mL}$

Analyser sample volume: $10 \mu \mathrm{L}$

Quality control: Standard laboratory QC procedures

Mailing acceptable to EU: Not known

Cut-off level: CE Marked for a user-defined cut-off

Limit of detection: $14 \mathrm{ng} / \mathrm{mL}$ buffer

Range Measuring range: $15-1000 \mathrm{ng} / \mathrm{mL}$.

Use in population screening: Italy [100] \& France

Recent pertinent scientific papers: [36]

Website URL: http://www.sentinel.it/uk/ 
Faecal sampling/collection system

Many factors influence the uptake and reliability of sample collection. Inappropriate implementation can result in grossly misleading results. No single collection method is supported by the literature; however, the following factors should be considered when selecting a device for taking samples in population screening:

- The distribution process should be reliable and reach all selected subjects.

- The laboratory should be able to unambiguously identify the subject ID on the test device perhaps using a suitable barcode.

- The laboratory should be able to check the manufacturer's device expiry date on each returned device.

- The instructions for using the device must be simple and clear.

- The device should to be simple and easy to use by the target population.

- The device should leave minimal opportunity for collection error.

- The device should facilitate consistency in the volume of sample collected.

- The device/instructions should discourage inappropriate repeat sampling into/onto the sample device.

- Misuse of the device by participants should not cause loss of sample buffer.

- The system should not be susceptible to interference from toilet bowl disinfectants, etc.

- The screening participant should be able to record the date of sample collection to ensure the laboratory can verify receipt within an acceptable sample stability period.

- The process used by the subject for returning the device should be simple, reliable, safe and, when appropriate, should meet EU postal regulations.

A local pilot study should be undertaken to ensure that the chosen device and associated distribution, sampling and labelling procedures are acceptable (Sect. 4.2.3, 4.2.4, 4.3.2.1, 4.3.3.4) $(\mathrm{VI}-\mathrm{A}) .^{\text {Rec }} 4.9$

\section{Laboratory organisation \\ - Number of laboratory sites}

Population screening necessitates the receipt, measurement and recording of thousands of tests each day. The samples should be analysed without delay to avoid further sample denaturation and avoid an increase in false negative results. Inter-laboratory analytical imprecision is well described and can be observed through established external quality assurance schemes. Improved consistency is achieved by adopting common analytical platforms, analytical and quality standards and shared staff training. The analysis needs to be reproducible across a screening population and therefore the number of analytical centres should be minimised with automated analytical systems utilised wherever possible and agreed common testing procedures adopted by each centre (Sect. 4.3.3.4) (VI-B). ${ }^{\text {Rec } 4.10}$

\section{- Laboratory staff}

All laboratories providing population screening should be led by a qualified clinical chemist who is trained and experienced in the techniques used for analysis and with clinical quality assurance procedures (Sect. 4.3.3.4) (VI-B). Rec 4.11

\section{- Laboratory accreditation and quality monitoring}

All laboratories providing screening services should be associated with a laboratory accredited to ISO 15189:2007 Medical laboratories - Particular requirements for quality and competence. The laboratories should perform internal quality control
(IQC) procedures and participate in an appropriate external quality assessment scheme (EQAS, Sect. 4.3.3.4) (VI-B). ${ }^{\text {Rec } 4.12}$

- Distribution of FOBT kits by mail

Distribution and receipt of FOBT kits using local postal services can be an effective means of reaching the designated population (Ch. 2 [76], Rec. 2.15, Sect. 2.5.1.1 and Sect. 4.4.3.4) (II-B). $\operatorname{Rec} 4.13$

\section{Identification of participants and test results}

Automated check protocols should be implemented to ensure correct identification of the screened population and complete and accurate recording of individual screening participation and test results (see Ch. 2 [76], Rec. 2.18, Sect 2.5.1.3) (VI-A). ${ }^{\text {Rec } 4.15}$

\section{Classification of test results}

Protocols should be implemented to ensure standardised and reliable classification of the test results (Ch. 2 [76], Rec 2.19, Sect. 2.5.1.3) (VI-A). ${ }^{\text {Rec } 4.16}$

\section{Quality assurance \\ - Quality assurance of gFOBT testing}

Whilst an immunochemical test is recommended, programmes that adopt a traditional guaiac test need to apply additional laboratory quality procedures. To minimise variability and error associated with visual test reading, including manual results input, the following procedures should be considered (Sect. 4.3.3.4) (VI-B): $\operatorname{Rec} 4.17$

- Use of appropriate temperature for artificial lighting and neutral-coloured walls in the reading laboratory;

- Use of a national laboratory training programme to prosper consistency of interpretation;

- A blinded internal QC check each day for each analyst prior to commencing testing;

- Adoption of a monitoring programme to identify operator related analytical performance (e.g. positivity variability and bias); and

$\checkmark$ Double entry of test results

\section{- Quality assurance of FIT testing}

Consistency in analytical performance must be assured by the adoption and application of rigorous quality assurance procedures. Manufacturer's instructions for use must be followed. Laboratories should perform daily checks of analytical accuracy and precision across the measurement range with particular emphasis at the selected cut-off limit. Rigorous procedures need to be agreed and adopted on how internal quality control data is interpreted and how the laboratory responds to unsatisfactory results. Performance data, both internal quality control and external quality assessment data, should be shared and reviewed by a quality assurance team working across the programme. Sufficient instrumentation should be available to avoid delays in analysis due to instrument failure or maintenance procedures (Sect. 4.3.3.4) (VI-B). ${ }^{\operatorname{Rec} 4.18}$

\section{- External quality assessment}

A European external quality assessment scheme should be developed to facilitate Europe-wide quality assurance of occult blood testing and enhance the reproducibility of testing within and between countries providing population screening (Sect. 4.3.3.4) (III-B). Rec $4.19^{-19}$

\section{- Outcome monitoring}

All aspects of laboratory performance in respect of the screening test should be part of a rigorous quality assurance system. Uptake, undelivered mail, time from collection to analysis, analytical performance (internal QC and external QA), positivity 
rates, lost \& spoilt kits and technical failure rate, technician performance variability and bias should each be subject to rigorous monitoring (Sect. 4.3.3.4) (VI-A). ${ }^{\text {Rec } 4.20}$

\section{- Quality of information}

The proportion of unacceptable tests received for measurement is influenced by the ease of use of the test kit and the quality of the instructions for use. This proportion should not exceed $3 \%$ of all kits received; less than $1 \%$ is desirable (see Ch. 3 [84], Rec. 3.9, Sect. 3.3.2) (III-A). Rec 4.21

\subsection{Clinical performance}

\subsubsection{Description of terms used to describe test effectiveness}

gFOBT screening has been proven to be effective in reducing colorectal cancer mortality [50]. In randomised trials the reduction in cause-specific mortality ranged from $15 \%$ [48] to $33 \%$ [77]. Such a large variance in mortality can be explained by test differences, different numbers of faecal samples, different intervals between invitation cycles (one-year or two-year) and different responses to invitation associated with the characteristics and composition of the population screened. The sensitivity and specificity quoted for a test will therefore be influenced both by the test's analytical characteristics and the context in which the test is used and evaluated.

gFOBTs come in two forms, the conventional form with normal sensitivity and the more sensitive variety, Hemoccult SENSA, in which the sample is hydrated before analysis. Hemoccult SENSA performs quite differently from the gFOBTs used in European trials $[48,63]$ and is both more sensitive and less specific. Comparison of the clinical performance of gFOBT and FIT is complex because FIT can have different levels of specificity and sensitivity, indeed they may have variable positive cut-off concentrations. Changes in cut-off concentrations result in different clinical performance characteristics.

Although only one population-based RCT has been described with FIT [121], the many published diagnostic accuracy studies provide information on the comparative ability of current tests to distinguish subjects with or without colorectal cancer and adenoma and can be considered an acceptable indication of the satisfactory performance of FIT in population screening [13].

Diagnostic accuracy studies have compared:

a) subjects performing one or both tests (gFOBT and FIT) and performing a total colonoscopy (or sigmoidoscopy) independently from the result of the test (cohort studies);

b) subjects performing one or both tests and undergoing colonoscopy if one or both tests are positive (cohort studies);

c) Diagnosis determined beforehand and the test performed subsequently (case-control studies); and

d) Different subjects performing different tests.

Colorectal cancer, large adenomas ( $\geq 10 \mathrm{~mm}$ ), high-risk adenomas (high-grade dysplasia, villous change, serrated histology or $\geq 3$ polyps), all adenomas (including small adenomas), alone or combined have been used as reference standards in the various studies.

The comparative clinical performance of the different tests has usually been based on the following indicators: Sensitivity, specificity, positive predictive value (PPV), false positive rate, likelihood ratio for a positive or a negative test which is derived from sensitivity and specificity (sensitivity/(1-specificity)) for + LR; (1-sensitivity)/specificity for-LR.
Table 4.5 Simple $2 \times 2$ contingency table.

\begin{tabular}{|lllll|}
\hline & & Disease Present & Disease Absent & \\
\hline & & + & - & Total \\
\hline Positive Test & + & $\mathrm{a}$ & $\mathrm{b}$ & $\mathrm{a}+\mathrm{b}$ \\
\hline Negative Test & - & $\mathrm{c}$ & $\mathrm{d}$ & $\mathrm{c}+\mathrm{d}$ \\
\hline & Total & $\mathrm{a}+\mathrm{c}$ & $\mathrm{b}+\mathrm{d}$ & \\
\hline
\end{tabular}

All of these parameters derive from the well-described $2 * 2$ table ( Table 4.5) Where, $a$ are true positive, $b$ are false positive, $c$ are false negative and $d$ are true negative.

Sensitivity $=a /(a+c)$

Specificity $=d /(b+d)$

$P P V=a /(a+b)$

"True" in true positive, is an abstract concept because in practice a reference standard must be adopted. For colorectal cancer screening, true is usually defined by the outcome of total colonoscopy, the best practical diagnostic procedure we have though it does not have a sensitivity of $100 \%$. In a clinical setting it is not always possible to perform a total colonoscopy on all subjects who have negative screening tests, so it is difficult to estimate the number of false negatives (c) and true negatives (d). The difficulty of estimating false negative has a great impact on sensitivity but much less so on specificity. In fact (c) is a number much lower than (d), so that the sum $c+d$ (i.e. the number of negatives to the test) is a small overestimate of $d$.

For sensitivity, (c) is a significant proportion of $(a+c)$, so that it is necessary to have a direct estimate of the number of false negatives. Very often this estimate is obtained by measurement of the interval cancers (i.e. the number of colorectal cancers that are diagnosed in subjects negative to the test during a defined interval of time). Clearly the reliability of the estimated number of false negatives will depend on the time interval, and that will increase as time elapses. It is therefore important when comparing estimates of sensitivity obtained in this way to verify that the time interval used is the same.

The ideal theoretical approach to estimating cancer-screening performance would be to obtain the disease status using a "gold-standard" method that is independent of the screening method. For colorectal cancer, the disease status is usually determined from a histological examination of biopsy specimens of those with positive test results, because it is not ethically acceptable to collect biopsies from all individuals undertaking a screening test. The sensitivity and specificity of screening test are therefore usually estimated using interval cancers. As initially described by Day [28] interval cancers will not include slow-growing cancers missed by the test and not evident between two screening events (therefore clinical sensitivity will be overestimated). Conversely, interval cancers will include fast-growing cancers not present at the time of the screening test, but developing during the interval period (thus underestimating clinical sensitivity). This limitation is common to all screening procedure evaluations.

Programme sensitivity is an estimate of sensitivity (i.e. the number of CRC detected/the number of cancers detected plus the number of interval cancers occurring in a certain interval of time) and is biased toward overdiagnosis of CRC (i.e. it estimates diagnosis of CRC that would never occur clinically). For this reason it is sometimes preferable to give an estimate of sensitivity based on the ratio between interval cancers (in a defined time period) and the number of cancers expected in the same period 
(more precisely, 1 -(interval cancers occurred in x years/expected cancers in $x$ years)). This estimate gives an idea of cancers anticipated by screening, and it is not affected by overdiagnosis.

It is also worth noting that from a practical point of view, the choice of the test (or combination of tests) is not based on clinical sensitivity and specificity but on the determination of detection rate (for cancer or adenomas) and its correlation with positivity being first correlated to sensitivity and later to specificity.

\subsubsection{Comparative clinical performance - gFOBT and FIT} Many studies comparing FIT and gFOBT have been performed in the last 8 years, and several systematic reviews of the literature have been undertaken more recently.

In 2007 Kerr published a systematic review by the Health Technology Assessment of New Zealand (NZHTA) which had the aim of identifying the international evidence for the clinical and cost effecttiveness of screening tests for colorectal cancer [61]. This review included all primary research published as full original reports and secondary research, systematic reviews and meta-analyses published since November 2004. It also included seven eligible primary research papers $[19,23,62,96,98,104,134]$ and five eligible secondary research papers; [10,24,26,91,133].

The review concluded that "there is limited definitive evidence regarding superior immunochemical FOBT performance over the guaiac tests. However, evidence from cross-sectional studies suggests that the immunochemical test HemeSelect (Beckman Coulter Inc. Fullerton, CA, USA) is comparable with or superior to guaiac testing... The conclusions on this topic should be revisited if further reliable evidence on the comparative performance of screening FOBTs becomes available".

A similar conclusion was reached in a systematic review commissioned by the UK NHS and undertaken by the Centre for Reviews and Dissemination of the University of York in 2007 [13] which examined the literature until 2004. The review included 59 studies. Thirty-nine evaluated gFOBTs, 33 evaluated FIT and one evaluated sequential FOBTs. It concluded that there was no clear evidence from direct or indirect comparisons to suggest that guaiac or immunochemical FOBTs performed better. However amongst FIT, Immudia-HemSp (now HemSp) appeared to be the most sensitive and specific.

In the four years since 2004, six studies comparing the performance of gFOBT and FIT have been published $[4,27,45,70,111$, 121]. Some further studies have investigated the accuracy of FIT which, although without a direct comparison with gFOBTs, confirmed the performance of FIT that was reported in the following studies $[15,68,82]$.

In Australia, Smith et al. [111] performed a paired comparison of an FIT (InSure) with a sensitive gFOBT (Hemoccult SENSA); 2351 asymptomatic and 161 symptomatic subjects were requested to perform both FOBTs. FIT returned a true-positive result significantly more often in cancer ( $\mathrm{n}=24 ; 87.5 \%$ vs $54.2 \%$ ) and in significant adenomas ( $n=61 ; 42.6 \%$ vs $23.0 \%$ ) while the false-positive rate for any neoplasia was marginally higher with the FIT than the gFOBT (3.4\% vs $2.5 \%$; $95 \% \mathrm{CI}$ of difference, $0-1.8 \%$ ): the PPV for cancer and significant adenomas was slightly better for FIT (41.9\% vs $40.4 \%$ ).

In Israel, Levi et al. [70] compared a gFOBT with an FIT (OC-Micro, now OC-Sensor) in a small number (151) of patients referred for colonoscopy either because of a positive gFOBT or because they were above average risk of colorectal cancer. Sensitivity, specificity, and PPV for significant colorectal neoplasia were 75\%, 34\% and $12 \%$, respectively, for gFOBT, and were $75 \%, 94 \%$ and $60 \%$ for FIT. For a positive gFOBT, 4 times more colonoscopies were needed to identify a significant neoplasm compared with FIT, and at more than 4 times greater cost.

In France, Guittet et al. [45] compared the performance of gFOBT and FIT (Immudia-HemSp (now HemSp)) among 10673 averagerisk persons aged 50-74 years. Colonoscopy was offered only if either FOBT was positive. The threshold for a positive FIT was varied between $20 \mathrm{ng} / \mathrm{mL}$ and $75 \mathrm{ng} / \mathrm{mL}$. Overall, the results depended on the adopted FIT threshold. At the lower threshold (20ng/ $\mathrm{mL}$ ), FIT detected 1.5 times as many cancers and nearly 2.6 times as many high-risk adenomas as gFOBT; however, it also increased the relative false-positive rates (2.17 times more frequent for each relevant lesion detected in FIT as compared with gFOBT). It is worth noting that at a threshold of $75 \mathrm{ng} / \mathrm{mL}$, FIT detected $90 \%$ more advanced neoplasms with a significant 33\% decrease in the false-positive risk. A further publication from this study [46] reported that the gain in sensitivity from using FIT vs gFOBT was proportional to the degree of blood loss from the lesion and its location. The benefits for cancer detection were restricted to lesion of the rectum.

In the US, Allison et al. [4] prospectively compared two types of FOBTs, a sensitive gFOBT (Hemoccult SENSA) and a manual FIT (FlexSure). A large number of patients (7394 subjects were eligible for the study) were requested to perform both tests. All patients positive for either FOBTs were invited to have a total colonoscopy, whereas all patients negative to FOBT were advised to have a sigmoidoscopy. All cancers occurring during the two years following the test were identified, so that it was possible to estimate the absolute sensitivity and specificity for detecting advanced neoplasms in the left colon within two years after the FOBT screening for the two tests administered separately and in combination. The sensitivity for detecting cancer was $81.8 \%$ (95\% CI, $47.8 \%$ to $96.8 \%$ ) for the FIT and 64.3\% (95\% CI, 35.6\% to $86.0 \%$ ) for the gFOBT. The sensitivity for detecting distal advanced adenomas was higher for gFOBT than for FIT 41.3\% (95 \% CI, $32.7 \%$ to $50.4 \%$ ) vs $29.5 \%$ ( $95 \%$ CI, $21.4 \%$ to $38.9 \%$ ). PPV was much higher for FIT than for gFOBT for distal cancer (5.2\% and $1.5 \%$ for FIT and gFOBT respectively) and for advanced adenomas (19.1 and $8.9 \%$ for FIT and gFOBT respectively). The authors concluded that FIT has high sensitivity and specificity for detecting left-sided colorectal cancer and that it may be a useful replacement for the gFOBT.

The study by Dancourt et al. [27] compared the performance of a 3-day gFOBT and 2-day FIT in 17215 subjects. For 1205 subjects who participated and had colonoscopy, the PPV for the guaiac and immunochemical test was respectively $5.9 \%$ vs $5.2 \%$ for cancer and $27.2 \%$ and $17.5 \%$ for adenoma.

The study by van Rossum et al. [121] represents a milestone in the comparison of gFOBT with FIT, being the first randomised trial in a population-based screening setting. A large number of people (20623) aged 50-75 years were randomised to either gFOBT (Hemoccult II, Beckman Coulter Inc. Fullerton, CA, USA) or FIT (OC-Sensor). For FIT, the standard cut-off of $100 \mathrm{ng} / \mathrm{mL}$ was used. FIT showed higher compliance than did gFOBTs (56.9\% vs $46.9 \%$ respectively $\mathrm{p}<0.01)$. The positivity rate was significantly higher in FIT compared with gFOBTs (5.0\% vs $2.4 \%$ respectively, $\mathrm{p}<0.01)$. Cancer or advanced adenomas were found, respectively, in 11 and 46 of gFOBTs and in 24 and 121 of FIT. The detection rate per 1000 examinations for cancer was 71\% higher in FIT compared with gFOBT; the detection rate per 1000 examinations for advanced adenomas was $106 \%$ higher in FIT as compared with gFOBT. The number-to-scope to find 1 cancer or 1 adenoma was 
comparable between the tests, with the PPV not statistically different. In conclusion, FIT compared with gFOBT demonstrated a higher detection rate with a similar PPV.

The results of these five studies are consistent with data from the first European screening programmes. The UK Pilot study adopted Hema-Screen, a conventional non-rehydrating gFOBT, using duplicate samples on 3 consecutive stools extended to 2 further sets of 3 stools if indicated. This UK pilot study gave a positivity rate during the first round of $1.9 \%$. The detection rates for cancer and neoplasia (cancer and advanced or non-advanced adenoma) were 1.62 in 1000 and 6.91 in 1000 respectively. The PPV for neoplasia was $46.9 \%$ in England and $47.3 \%$ in Scotland [117].

In Italy, a 1-day single sample FIT biennial test with positivity cutoff at $100 \mathrm{ng} / \mathrm{mL}$ is used in the regional colorectal cancer programmes. The paper by Zorzi that described Italian screening programmes showed a quite different outcome to the UK Pilot study [137]. The positivity rate was relatively high, 5.3\% during the first round, the detection rate for cancer was 3.1 in 1000 (almost two times the UK figure) and the detection rate for adenoma was 24.7 in 1000 (more than three times the UK result). The PPV for neoplasia was slightly higher than that observed in UK pilot study (54\% vs $46.9 \%$ ) [117]. The Italian programme had adopted a more sensitive (but less specific) strategy compared with the UK.

Hol et al. [51] recently reported a randomised comparison of gFOBT (Hemoccult II) and FIT (OC-Sensor) in a population-based trial in the southwest Netherlands (age 50 - 74 years). For gFOBT, any 1 of 6 windows collected from 3 stools was designated positive and for FIT a single result above a cut-off concentration of 50 $\mathrm{ng} / \mathrm{mL}$ was designated positive. Test kits were all distributed and returned by mail. Participants with positive results received colonoscopy. gFOBT positivity was $2.8 \%$, and FIT positivity was $8.1 \%$ at a cut-off of $50 \mathrm{ng} / \mathrm{mL}, 5.7 \%$ at $75 \mathrm{ng} / \mathrm{mL}, 4.8 \%$ at $100 \mathrm{ng} / \mathrm{mL}$ and $4.0 \%$ at $150 \mathrm{ng} / \mathrm{mL}$. At an FIT cut-off concentration of $75 \mathrm{ng} / \mathrm{mL}$, the detection rate for advanced neoplasia was $2 \times$ higher than that by gFOBT and was considered to be the optimum cut-off and balance between detection rate and positivity.

\subsubsection{Optimising clinical performance using test cut-off limits \& algorithms}

\subsubsection{Cut-off limits}

Until recently it has not been possible to adjust the analytical sensitivity of FOBT tests. This is still not possible for existing gFOBTs, with the exception of the simple adjunct of hydrating the specimen before testing with Hemoccult SENSA. With Hemoccult SENSA, hydration increases test sensitivity at the expense of specificity, thereby increasing the false-positive rate $[77,93]$. Hemoccult and Hemoccult SENSA have been compared in two large studies [77]. As a result of rehydration, the rate of positive results increased more than fourfold, from 2.4 to $9.8 \%$. Sensitivity increased from $80.8 \%$ to $92.2 \%$ while both specificity and PPV decreased (specificity: $90.4 \%$ rehydrated and $97.7 \%$ non-rehydrated. PPV: 2.2 rehydrated and 5.6 non-rehydrated). In the study by Levin, Hess \& Johnson [71] the positivity rates were $5 \%$ and $14.6 \%$ and PPV $14 \%$ and $7 \%$ respectively for the non-rehydrated and the rehydrated. Rehydration using Hemoccult SENSA increases clinical sensitivity and decreases specificity and PPV. The high positivity rate of this approach renders it unsuitable for population screening.

With FIT that provide a numeric result, it is possible to adjust the cut-off limit to obtain an acceptable compromise between clini- cal sensitivity and specificity. This manipulation can provide an adequate detection rate from an acceptable cohort of subjects invited for colonoscopy. Several recent papers have addressed the issue of modifying the faecal haemoglobin cut-off limit of FIT including the following studies $[14,16,17,67,87,99,109,120,122]$. The data are summarised in $\odot$ Table 4.6. By increasing the positive cut-off limit, the test sensitivity and positivity rate decreases and specificity and PPVs for colorectal cancer detection increase. It must be appreciated that these studies used different commercial products with different analytical characteristics, and therefore simple comparisons can be misleading.

Chen found an analytical cut-off limit range of $100-150 \mathrm{ng} / \mathrm{mL}$ faecal haemoglobin in an FIT to provide an acceptable balance between sensitivity and specificity $[17,87]$. Increasing the cut-off limit to $300 \mathrm{ng} / \mathrm{mL}$ brought an increase in specificity that was small for the corresponding decrease in sensitivity and detection of cancers. A recent study by van Rossum of 615750 - 75-year-old Dutch participants and using a single OC-Sensor collection and OC-Micro analyser concluded that dropping from the standard $100 \mathrm{ng} / \mathrm{mL}$ cut-off to $75 \mathrm{ng} / \mathrm{mL}$ brought 'optimal' results and may be recommended for population screening in the Netherlands [120]. This study also concluded that where colonoscopy capacity is insufficient, a cut-off up to $200 \mathrm{ng} / \mathrm{mL}$ would result in minimal false negatives for cancer although more for advanced adenoma. Policy makers are faced with an arbitrary decision based on the balance between missed cancers/advanced adenomas and the cost of colonoscopy.

\subsubsection{Number of stool specimens}

Several studies have now examined the influence of the number of samples used for testing on clinical sensitivity and specificity. Allison takes any positive result from 3 stool samples measured using FlexSure OBT as an indication for referral and shows higher sensitivity for cancer than studies using single stool samples [4]. Unsurprisingly other studies show agreement with that conclusion $[5,42,85,86,96,112,129]$. Nakama et al. using MonoHaem, showed sensitivities of $89 \%$ for cancer with 3 stools compared with $56 \%$ for a single stool [85].

Using HemSp, Morikawa showed low sensitivity for cancer using a single-day sample [82]. Rozen et al. [99] used 3 stools for the OC-Sensor which contrasts with 2-day samples used in Japan [86] and 1-day biennial testing performed in Italy [14]. The relative insensitivity in the Italian study to lesions in the proximal bowel (16.3 vs $30.7 \%$ ) raises further doubts about the use of a single-day sample. In a study using OC-Sensor in an at-risk population, Levi et al. [68] took numeric measurements from three samples and used the highest concentration of the three as the discriminating factor. Recent studies have taken the average concentration from 2 stool measurements as the discriminating parameter, an approach that reduces the positivity rate.

The use of different cut-off limits and different numbers of stool samples illustrates how programme algorithms can manipulate clinical sensitivities and specificities for the lesions of interest. Chen describes the use of a cost-effectiveness model as a method of determining the optimal cut-off concentration for an FIT [17]. In the study by Levi et al. [68] using an FIT OC-Micro, a scatter plot of 2 consecutive samples showed that of those with cancer or adenomas, 21 of 91 had elevated or markedly elevated faecal blood in one sample but were normal in the other. This is further evidence of intermittent or variable bleeding, sample heterogeneity or poor sample technique that will reduce clinical sensitivity. Imperiale [55] commenting on the paper by Levi in his editor- 
Table 4.6 Comparison of clinical performance at different cut-off concentrations

\begin{tabular}{|c|c|c|c|c|c|c|c|c|}
\hline Study & $\begin{array}{l}\text { Faecal Hb } \\
\text { cut-off (ng/mL) }\end{array}$ & Japan [86] & $\begin{array}{l}\text { Italy } \\
\text { (OC-Hemodia) } \\
{[16]}\end{array}$ & $\begin{array}{l}\text { Italy } \\
\text { (Latex agglutination) } \\
{[14]}\end{array}$ & France [66] & $\begin{array}{l}\text { Taiwan } \\
\text { [72] }\end{array}$ & $\begin{array}{l}\text { Israel } \\
{[99,122]}\end{array}$ & $\begin{array}{l}\text { Germany } \\
\text { [109] }\end{array}$ \\
\hline \multirow[t]{7}{*}{ Test Positivity (\%) } & 20 & - & - & - & 5.8 & - & - & - \\
\hline & 50 & 6.5 & - & - & 3.1 & - & - & - \\
\hline & 75 & - & - & - & 2.0 & - & - & - \\
\hline & 100 & - & 3.5 & 4.2 & - & 5.5 & - & - \\
\hline & 150 & 4.1 & 2.5 & 3.0 & - & - & - & - \\
\hline & 200 & & 2.0 & 2.3 & - & - & - & - \\
\hline & 300 & 3.3 & - & - & - & - & - & - \\
\hline \multirow[t]{7}{*}{ Test Sensitivity (\%) } & 20 & - & - & & 85.0 & - & - & - \\
\hline & 50 & 89 & - & & $68.0-83.0$ & 81.5 & 79.4 & - \\
\hline & 75 & - & - & & $61.0-81.0$ & - & 76.5 & - \\
\hline & 100 & - & 84.0 & & - & 81.5 & 76.5 & - \\
\hline & 150 & 81 & 78.9 & & - & 69.2 & 70.6 & 87 \\
\hline & 200 & - & 73.4 & & - & 64.6 & 64.7 & 83 \\
\hline & 300 & 56 & - & & - & - & - & 78 \\
\hline \multirow[t]{7}{*}{ Test Specificity (\%) } & 20 & - & - & & 94.0 & & - & - \\
\hline & 50 & 94 & - & & 97.0 & & 89.7 & - \\
\hline & 75 & - & - & & 98.0 & & 93.3 & - \\
\hline & 100 & - & 97.2 & & - & & 95.3 & - \\
\hline & 150 & 96 & 97.2 & & - & & 95.9 & - \\
\hline & 200 & - & 97.2 & & - & & 96.3 & - \\
\hline & 300 & 97 & - & & - & & - & - \\
\hline \multirow[t]{7}{*}{ PPV for CRC (\%) } & 20 & - & - & - & 6.0 & & - & - \\
\hline & 50 & 8.6 & - & - & 9.0 & & 36.0 & - \\
\hline & 75 & - & - & - & 13.0 & & 45.6 & - \\
\hline & 100 & - & 8.8 & 9.0 & - & & 54.2 & - \\
\hline & 150 & 12.6 & 11.5 & 11.6 & - & & 54.5 & - \\
\hline & 200 & - & 13.9 & 13.4 & - & & 56.4 & - \\
\hline & 300 & 10.8 & - & - & - & & - & - \\
\hline
\end{tabular}

ial in Annals of Internal Medicine [68], speculated that concentration-related clinical sensitivity and specificity could be used to determine post-test risk. If risk was related to subject age or sex, this would provide more sophisticated criteria for colonoscopy referral than is currently used.

Guittet et al. [47], using a cut-off concentration of $20 \mathrm{ng} / \mathrm{mL}$, reviewed the relative effectiveness of using one sample, one positive from two samples, two positives from two samples or a mean positive from two samples all measured using the MagStream FIT. The study concluded that for any sensitivity the mean of two results provided the highest specificity, and at any positivity it provided the highest sensitivity and specificity. It also concluded that one positive from a single specimen was better than one from two specimens and the cut-off should be adjusted to provide an acceptable positivity rate.

A recent paper by Grazzini et al. [41] looked at the clinical outcome of biennial population screening in 20596 residents of Northern and Central Italy aged 50-69 years. The study uses OC-Sensor and compares outcomes from strategies using different cut-off limits $(80,100$ and $120 \mathrm{ng} / \mathrm{mL})$, one or two samples and referral criteria based on either one positive or two positive results. No strategy is singled out as preferable, although some show limited benefit. Generally, those strategies resulting in more colonoscopy referrals increase the detection rate, particularly for adenomas, decrease the PPV and cost more. At the annual Digestive Diseases Week conference in 2010 van Roon et al. [119] illustrated the relationship between positivity rate, detection rate, cut-off limits, the number of samples measured and the use of different algorithms for combining the results. For positivity rates between $4 \%$ and $9 \%$ the user can obtain similar clinical outcomes by changing the cut-off with either one or two samples. The dilemma for a population-screening programme is where to draw the line between detection rates, cost and the inconvenience and morbidity associated with colonoscopy. The study showed no significant reduction in uptake for the twosample strategy, but it did require the samples to be stored in a refrigerator. The choice is likely to be influenced greatly by both financial and logistical considerations.

\subsubsection{Sequential testing}

Two consecutive diagnostic accuracy studies conducted in Scotland as part of the UK pilot screening study investigated whether testing individuals with positive gFOBT tests using an FIT could be more effective in selecting those who should receive colonoscopy $[37,38]$. In both studies the two-tier approach gave very high sensitivities of $95-96 \%$ with a negative carrying a less than $1 \%$ chance of invasive cancer. The odds ratio for FIT positive subjects of having cancer was 7.75 (95\% CI, 1.84-31.4).

A Chinese study [73] of 324 subjects who had colonoscopy (mean age $53.5 \pm 15.3$ ) showed that a FIT following a positive gFOBT had a better specificity for colon cancer detection than gFOBT ( $94.2 \%$ vs $75.5 \%$ ), and with similar sensitivity (93.8\% and $95.9 \%$ vs $95.9 \%$, p $>0.05)$.

In a multicentre comparison using different FOBT tests on 554 patients referred for colonoscopy (mean age $59.8 \pm 11.7$ ), a combination test with a highly sensitive gFOBT (Hemoccult SENSA) and 
an FIT (FlexSure (FS) or HemeSelect (HS), Beckman Coulter Inc. Fullerton, CA, USA) showed slightly reduced sensitivity but significantly fewer false-positive tests than any single test [42]. The specificity of SENSA/FS (95.7\%, p=0.03) and SENSA/HS (95.2\%, $\mathrm{p}=0.07$ ) for the detection of colorectal cancer were each greater than that of any individual test.

\subsubsection{Participation rate and choice of test}

Factors that influence participation rate (uptake) are addressed in Chapter 2 [76] (Sect. 2.4, 2.5.1.1 and 2.5.1.2). Whilst many studies have reported the effect on compliance of different test devices and sampling permutations, some of these are contradictory and many reflect local circumstances. Whilst the analytical method, gFOBT vs FIT, will not directly influence compliance, the influence of test method on the method of sampling, the number of samples required, a requirement for dietary restriction and the improved clinical outcome will all have a bearing on uptake. The magnitude of the influence will depend on local circumstances. Well-conducted randomised trials have clearly demonstrated that better compliance can be achieved using current FIT than with gFOBTs, but the major influencing factor(s) remain a matter of speculation. In his recent paper Grazzini makes the important observation that, in a biennial screening programme looking for a slow growing adenoma, greater compliance over the long term might be more important than a higher detection rate on a single screen [41].

\subsubsection{Recommendations Screening algorithm: \\ - Sample and test numbers}

Few studies have examined the number of stool specimens necessary to optimise the diagnostic performance of FOBT. Consideration should be given to using more than one specimen together with criteria for assigning positivity which together provide a referral rate that is clinically, logistically and financially appropriate to the screening programme. The clinical sensitivity and specificity of testing can be modified depending on how the test data are used. Guaiac-based tests typically use 3 stools, but an algorithm using additional tests can be used to adjust clinical sensitivity and specificity (Sect. 4.4.3.2, 4.4.3.1) (III-C). ${ }^{\text {Rec } 4.5}$

\section{- Determining test positivity}

The choice of a cut-off concentration to be used in an immunochemical test to discriminate between a positive and negative result will depend on the test device chosen, the number of samples used and the algorithm adopted to integrate the individual test results. Whilst an increasing number of studies are reporting the experience of different algorithms, local conditions, including the effect on sample stability of transport conditions, preclude a simple prescribed algorithm at this time. Adoption of a test device and the selection of a cut-off concentration should follow a local pilot study to ensure that the chosen test, test algorithm and transport arrangements work together to provide a positivity rate that is clinically, logistically and financially acceptable (Sect. 4.4.3.1, 4.4.3.2) (VI-A). ${ }^{\text {Rec 4.6 }}$

Maximisation of uptake - Influencing factors associated with the test kit
The choice of the test kit must be influenced by factors that enhance accessibility and uptake (see below and Sect. 4.2.3 and 4.2.4; see also Ch. 2 [76], Rec. 2.14, Sect. 2.5.1.1) (II-A). ${ }^{\text {Rec } 4.14}$

\section{- Dietary restrictions}

In order to enhance participation in screening, test kits should not require dietary restrictions (Ch. 2 [76], Rec. 2.17, Sect. 2.5.1.1; 4.3.2.1 and 4.3.2.3) (II-A).

\section{Kit design}

The design of a test kit should make it acceptable to the target population (see Ch. 2 [76], Rec. 2.14, Sect. 2.5.1.1, 4.2.3 and 4.2.4) (II-A).

- Simple and clear instructions

A clear and simple instruction sheet should be provided with the test kit (Ch. 2 [76], Rec. 2.16, Sect. 2.5.1.1; Sect. 4.2.3 and 4.2.4) (V-A).

\subsection{Conclusions}

\section{$\nabla$}

Although it is difficult to draw simple conclusions from the variety of different tests and study settings, we can conclude that FIT, in comparison with gFOBT:

- Has no need for dietary restriction;

- Has a major problem with sample instability, and collected samples preferably should be kept cool and returned immediately for analysis;

- Provides a greater participation rate than gFOBT;

- Needs a smaller number of stool samples than gFOBT;

- Shows a greater relative sensitivity than gFOBT;

- Shows a greater sensitivity for the detection of advanced adenomas than gFOBT;

- Has a higher recall rate than most gFOBTs;

- Has a PPV similar to those obtained with most gFOBTs;

- Provides an opportunity of using a numeric threshold to find the most appropriate balance between sensitivity and specificity (i.e. between detection rate and positivity to the test); and

- Allows the opportunity to balance recall and detection rates providing each country with the tools to implement a colorectal cancer screening programme that will meet local healthcare expectations within available resources.

In a multidisciplinary process, wide consensus has been achieved on a comprehensive package of evidence-based recommendations for faecal occult blood testing in colorectal cancer screening and diagnosis. Following these recommendations has the potential to enhance the control of colorectal cancer in Europe and elsewhere through improvement in the quality and effectiveness of the screening process that extends from systematic invitation to management of screen-detected cases.

\section{Acknowledgements \\ $\nabla$}

The comments and suggestions received from the following reviewers are gratefully acknowledged:

Jean Faivre, France, Hiroshi Saito, Japan, and Steve Smith, United Kingdom

The comments and suggestions received from consultation of the European Cancer Network are gratefully acknowledged.

The production of the Guidelines was supported by the European Union through the EU Public Health Programme, (grant agreement no.2005317: Development of European Guidelines for Quality Assurance of Colorectal Cancer Screening). Partner insti- 
tutions: Oxford University Cancer Screening Research Unit, Cancer Epidemiology Unit, University of Oxford, Oxford, United Kingdom; Unit of Cancer Epidemiology, Centre for Cancer Epidemiology and Prevention (CPO) and S. Giovanni University Hospital, Turin, Italy; Public Association for Healthy People, Budapest, Hungary; European Cancer Patient Coalition (ECPC), Utrecht, Netherlands; Quality Assurance Group, Section of Early Detection and Prevention, International Agency for Research on Cancer, Lyon, France.

Financial support was also received through the Public Affairs Committee of the United European Gastroenterology Federation, and from a cooperative agreement between the American Cancer Society and the Division of Cancer Prevention and Control at the Centers for Disease Control and Prevention.

\section{Disclaimer}

The views expressed in this document are those of the authors. Neither the European Commission nor any person acting on its behalf can be held responsible for any use that may be made of the information in this document.

\section{Competing interests: No competing interests reported}

\section{References}

1 Achord JL. Positive Hemoccult reactions after oral iron: true or false? Gastroenterology 1983; 84: 670-672

2 Ahlquist DA, McGill DB, Schwartz S et al. HemoQuant, a new quantitative assay for fecal hemoglobin. Comparison with Hemoccult. Ann Intern Med 1984; 101: 297-302

3 Ahlquist DA, McGill DB, Schwartz $S$ et al. Fecal blood levels in health and disease. A study using HemoQuant. N Engl J Med 1985; 312: $1422-1428$

4 Allison JE, Sakoda LC, Levin TR et al. Screening for colorectal neoplasms with new fecal occult blood tests: update on performance characteristics. J Natl Cancer Inst 2007; 99: 1462 -1470

5 Allison JE, Tekawa IS, Ransom LJ et al. A comparison of fecal occultblood tests for colorectal-cancer screening. N Engl J Med 1996; 334 : 155-159

6 Anderson GD, Yuellig TR, Krone RE et al. An investigation into the effects of oral iron supplementation on in vivo Hemoccult stool testing. Am J Gastroenterol 1990; 85: 558-561

7 Atkin W, Valori R, Kuipers EJ et al. European guidelines for quality assurance in colorectal cancer screening and diagnosis. First edition. Colonoscopic surveillance following adenoma removal. Endoscopy 2012; 44: SE151 - SE163

8 Austoker J, Giordano L, Hewitson P et al. European guidelines for quality assurance in colorectal cancer screening and diagnosis. First edition. Communication. Endoscopy 2012; 44: SE106 - SE115

9 Screening Retest Fact Sheet. Australian Government DOHAA; 2009: http://www.cancerscreening.gov.au/internet/screening/publishing. nsf/Content/bw-retest Accessed 11/2/2012

10 Australian Health Technology Advisory Committee (AHTAC). Colorectal Cancer Screening. Canberra: Commonwealth Department of Health and Family Services; 1997

11 Bennitt W, Burtonwood C, Halloran S. Four faecal occult blood test kits. [monograph on the Internet]. London (UK): NHS Purchasing and Supply Agency, Centre for Evidence-based Purchasing; 2006. Report No. 05110. http://nhscep.useconnect.co.uk/CEPProducts/catalogue. aspx [Accessed 4/07/2012]

12 Bini EJ, Rajapaksa RC, Weinshel EH. Positive predictive value of fecal occult blood testing in persons taking warfarin. Am J Gastroenterol 2005; 100: 1586 - 1592

13 Burch JA, Soares-Weiser K, St John DJ et al. Diagnostic accuracy of faecal occult blood tests used in screening for colorectal cancer: a systematic review. J Med Screen 2007; 14: 132 - 137
14 Castiglione G, Grazzini G, Miccinesi G et al. Basic variables at different positivity thresholds of a quantitative immunochemical test for faecal occult blood. J Med Screen 2002; 9: 99-103

15 Castiglione G, Visioli CB, Ciatto $S$ et al. Sensitivity of latex agglutination faecal occult blood test in the Florence District population-based colorectal cancer screening programme. Br J Cancer 2007; 96: $1750-1754$

16 Castiglione G, Zappa M, Grazzini G et al. Screening for colorectal cancer by faecal occult blood test: comparison of immunochemical tests. J Med Screen 2000; 7: 35-37

17 Chen LS, Liao CS, Chang SH et al. Cost-effectiveness analysis for determining optimal cut-off of immunochemical faecal occult blood test for population-based colorectal cancer screening (KCIS 16). J Med Screen 2007; 14: 191 - 199

18 Chen WD, Han ZJ, Skoletsky J et al. Detection in fecal DNA of colon cancer-specific methylation of the nonexpressed vimentin gene. J Natl Cancer Inst 2005; 97: 1124-1132

19 Cheng TI, Wong JM, Hong CF et al. Colorectal cancer screening in asymptomaic adults: comparison of colonoscopy, sigmoidoscopy and fecal occult blood tests. J Formos Med Assoc 2002; 101: 685-690

20 Ciatto S, Martinelli F, Castiglione G et al. Association of FOBT-assessed faecal $\mathrm{Hb}$ content with colonic lesions detected in the Florence screening programme. Br J Cancer 2007; 96: 218-221

21 Clarke P, Jack F, Carey FA et al. Medications with anticoagulant properties increase the likelihood of a negative colonoscopy in faecal occult blood test population screening. Colorectal Dis 2006; 8: 389-392

22 Cole SR, Young GP. Effect of dietary restriction on participation in faecal occult blood test screening for colorectal cancer. Med J Aust 2001; 175: $195-198$

23 Cole SR, Young GP, Esterman A et al. A randomised trial of the impact of new faecal haemoglobin test technologies on population participation in screening for colorectal cancer. J Med Screen 2003; 10: 117122

24 Conseil d'Évaluation des Technologies de la Santé du Quebec. Colorectal cancer screening. Montreal: Conseil d'Évaluation des Technologies de la Santé du Quebec; 2000

25 Council Recommendation of 2 December 2003 on cancer screening (2003/878/EC). Off J Eur Union 2003: 34-38

26 Craven 0 . Screening for colorectal cancer using the faecal occult blood test: a critical literature review. Eur J Oncol Nurs 2001; 5: 234-243

27 Dancourt V, Lejeune C, Lepage $C$ et al. Immunochemical faecal occult blood tests are superior to guaiac-based tests for the detection of colorectal neoplasms. Eur J Cancer 2008; 44: 2254-2258

28 Day NE. Estimating the sensitivity of a screening test. J Epidemiol Community Health 1985; 39: $364-366$

29 Ellis RJ, Wilson S, Holder RL et al. Different faecal sampling methods alter the acceptability of faecal occult blood testing: a cross sectional community survey. Eur J Cancer 2007; 43: 1437-1444

30 Faure H, Exbrayat C, Winckel $P$ et al. Moisture content of Hemoccult slides influences test sensitivity. Eur J Gastroenterol Hepatol 2003; 15: $1111-1114$

31 Federici A, Giorgi RP, Borgia P et al. The immunochemical faecal occult blood test leads to higher compliance than the guaiac for colorectal cancer screening programmes: a cluster randomized controlled trial. J Med Screen 2005; 12: 83-88

32 Fenocchi E, Martinez L, Tolve J et al. Screening for colorectal cancer in Uruguay with an immunochemical faecal occult blood test. Eur J Cancer Prev 2006; 15: 384-390

33 Ferlay J, Shin HR, Bray F et al. GLOBOCAN 2008 v1.2, Cancer Incidence and Mortality Worldwide: IARC CancerBase No. 10 [Internet]. Lyon, France: International Agency for Research on Cancer; 2010: Available from: http://globocan.iarc.fr Accessed on 05/04/2012

34 Fludger S, Turner AM, Harvey RF et al. Controlled prospective study of faecal occult blood screening for colorectal cancer in Bury, black pudding capital of the world. BMJ 2002; 325: 1444-1445

35 Fraser CG. Faecal occult blood tests - eliminate, enhance or update? Ann Clin Biochem 2008; 45: 117-121

36 Fraser CG, Mathew CM, McKay K et al. Automated immunochemical quantitation of haemoglobin in faeces collected on cards for screening for colorectal cancer. Gut 2008; 57: 1256 - 1260

37 Fraser CG, Mathew CM, Mowat NA et al. Evaluation of a card collection-based faecal immunochemical test in screening for colorectal cancer using a two-tier reflex approach. Gut 2007; 56: 1415-1418 
38 Fraser CG, Matthew CM, Mowat NA et al. Immunochemical testing of individuals positive for guaiac faecal occult blood test in a screening programme for colorectal cancer: an observational study. Lancet Oncol 2006; 7: $127-131$

39 Garrick DP, Close JR, McMurray W. Detection of occult blood in faeces. Lancet 1977; 2: 820-821

40 Grazzini G, Ventura L, Zappa $M$ et al. Influence of seasonal variations in ambient temperatures on performance of immunochemical faecal occult blood test for colorectal cancer screening: observational study from the Florence district. Gut 2010; 59: 1511 - 1515

41 Grazzini $G$, Visioli $C B$, Zorzi $M$ et al. Immunochemical faecal occult blood test: number of samples and positivity cutoff. What is the best strategy for colorectal cancer screening? Br J Cancer 2009; 100: 259 265

42 Greenberg PD, Bertario L, Gnauck $R$ et al. A prospective multicenter evaluation of new fecal occult blood tests in patients undergoing colonoscopy. Am J Gastroenterol 2000; 95: 1331 - 1338

43 Greenberg PD, Cello JP, Rockey DC. Relationship of low-dose aspirin to GI injury and occult bleeding: a pilot study. Gastrointest Endosc 1999; 50: 618-622

44 Greenwald $B$. A pilot study evaluating two alternate methods of stool collection for the fecal occult blood test. Medsurg Nurs 2006; 15: 89 94

45 Guittet L, Bouvier V, Mariotte $N$ et al. Comparison of a guaiac based and an immunochemical faecal occult blood test in screening for colorectal cancer in a general average risk population. Gut 2007; 56 : $210-214$

46 Guittet L, Bouvier V, Mariotte $N$ et al. Comparison of a guaiac and an immunochemical faecal occult blood test for the detection of colonic lesions according to lesion type and location. Br J Cancer 2009; 100: $1230-1235$

47 Guittet L, Bouvier V, Mariotte N et al. Performance of immunochemical faecal occult blood test in colorectal cancer screening in average-risk population according to positivity threshold and number of samples. Int J Cancer 2009; 125: 1127 - 1133

48 Hardcastle JD, Chamberlain JO, Robinson $\mathrm{MH}$ et al. Randomised controlled trial of faecal-occult-blood screening for colorectal cancer Lancet 1996; 348: 1472 - 1477

49 Haug $U$, Brenner $H$. New stool tests for colorectal cancer screening: a systematic review focusing on performance characteristics and practicalness. Int J Cancer 2005; 117: 169-176

50 Hewitson P, Glasziou P, Irwig $L$ et al. Screening for colorectal cancer using the faecal occult blood test, Hemoccult. Cochrane Database Syst Rev 2007: CD001216

$51 \mathrm{Hol}$ L, Wilschut JA, van Ballegooijen $M$ et al. Screening for colorectal cancer: random comparison of guaiac and immunochemical faecal occult blood testing at different cut-off levels. Br J Cancer 2009; 100: $1103-1110$

52 IARC. Chemical Agents and Related Occupations. 2012: IARC Monographs on the Evaluation of Carcinogenic Risks to Humans Vol. 100F.

53 Illingworth DG. Influence of diet on occult blood tests. Gut 1965; 6: $595-598$

54 Imafuku Y, Nagai T, Yoshida $H$. The effect of toilet sanitizers and detergents on immunological occult blood tests. Clin Chim Acta 1996; 253: $51-59$

55 Imperiale TF. Quantitative immunochemical fecal occult blood tests: is it time to go back to the future? Ann Intern Med 2007; 146: 309 311

56 Imperiale TF, Ransohoff DF, Itzkowitz SH et al. Fecal DNA versus fecal occult blood for colorectal-cancer screening in an average-risk population. N Engl J Med 2004; 351: $2704-2714$

57 Jaffe RM, Kasten B, Young DS et al. False-negative stool occult blood tests caused by ingestion of ascorbic acid (vitamin $C$ ). Ann Intern Med 1975; 83: 824-826

58 Jaffe RM, Zierdt W. A new occult blood test not subject to false-negative results from reducing substances. J Lab Clin Med 1979; 93: 879 886

59 Kahi CJ, Imperiale TF. Do aspirin and nonsteroidal anti-inflammatory drugs cause false-positive fecal occult blood test results? A prospective study in a cohort of veterans. Am J Med 2004; 117: 837-841

60 Keong $\mathrm{CH}$. CITES as a tool in combatting illegal logging. International Forestry Review 2009; 9: 805 - 810

61 Kerr J, Day P, Broadstock $M$ et al. Systematic review of the effectiveness of population screening for colorectal cancer. N Z Med J 2007; 120: U2629
62 Ko CW, Dominitz JA, Nguyen TD. Fecal occult blood testing in a general medical clinic: comparison between guaiac-based and immunochemical-based tests. Am J Med 2003; 115: 111 -114

63 Kronborg 0 , Fenger C, Olsen J et al. Randomised study of screening for colorectal cancer with faecal-occult-blood test. Lancet 1996; 348: 1467 - 1471

64 Lamph SA, Bennitt WE, Brannon CR et al. Evaluation report: Immunochemical faecal occult blood tests. Guildford: Centre for Evidencebased Purchasing; 2009: Report No. CEP 09042. http://www.cancerscreening.nhs.uk/bowel/ifobt.pdf [Accessed 28/07/2012]

65 Landsorp-Vogelaar I, von Karsa L. European guidelines for quality assurance in colorectal cancer screening and diagnosis. First edition. Introduction. Endoscopy 2012; 44: SE15 - SE30

66 Launoy $G$, Berchi $C$. [Advantage of immunochemical fecal occult blood test in screening for colorectal cancer]. Bull Cancer 2005; 92: 885 890

67 Launoy GD, Bertrand HJ, Berchi C et al. Evaluation of an immunochemical fecal occult blood test with automated reading in screening for colorectal cancer in a general average-risk population. Int J Cancer 2005; $115: 493-496$

68 Levi $Z$, Rozen P, Hazazi $R$ et al. A quantitative immunochemical fecal occult blood test for colorectal neoplasia. Ann Intern Med 2007; 146: $244-255$

69 Levi Z, Rozen P, Hazazi $R$ et al. Sensitivity, but not specificity, of a quantitative immunochemical fecal occult blood test for neoplasia is slightly increased by the use of low-dose aspirin, NSAIDs, and anticoagulants. Am J Gastroenterol 2009; 104: 933 - 938

70 Levi $Z$, Rozen P, Hazazi R et al. Can quantification of faecal occult blood predetermine the need for colonoscopy in patients at risk for nonsyndromic familial colorectal cancer? Aliment Pharmacol Ther 2006 24: $1475-1481$

71 Levin B, Hess K, Johnson C. Screening for colorectal cancer. A comparison of 3 fecal occult blood tests. Arch Intern Med 1997; 157: 970 976

$72 \mathrm{Li}$ CM, Shiu MN, Chia SL et al. Factors associated with referral compliance of abnormal immunochemical faecal occult blood test. J Med Screen 2007: 14: 186-190

$73 \mathrm{Li} \mathrm{S}$, Wang $\mathrm{H}, \mathrm{Hu}$ J et al. New immunochemical fecal occult blood test with two-consecutive stool sample testing is a cost-effective approach for colon cancer screening: results of a prospective multicenter study in Chinese patients. Int J Cancer 2006; 118: 3078 - 3083

74 Lifton LJ, Kreiser J. False-positive stool occult blood tests caused by iron preparations. A controlled study and review of literature. Gastroenterology 1982; 83: 860-863

75 Macrae FA, St John DJ. Relationship between patterns of bleeding and Hemoccult sensitivity in patients with colorectal cancers or adenomas. Gastroenterology 1982; 82: 891 - 898

76 Malila N, Senore C, Armaroli P. European guidelines for quality assurance in colorectal cancer screening and diagnosis. First edition. Organisation. Endoscopy 2012; 44: SE31 - SE48

77 Mandel JS, Bond JH, Church TR et al. Reducing mortality from colorectal cancer by screening for fecal occult blood. Minnesota Colon Cancer Control Study. N Engl J Med 1993; 328: 1365-1371

78 Minozzi S, Armaroli P, Banzi R et al. European guidelines for quality assurance in colorectal cancer screening and diagnosis. First edition. Appendix 1: Systematic evidence review. 2010: http://bookshop. europa.eu/en/european-guidelines-for-quality-assurance-in-colorectal-cancer-screening-and-diagnosis-pbND3210390/ [Accessed 11/2/ 2012]

79 Minozzi S, Armaroli P, Segnan N. European guidelines for quality assurance in colorectal cancer screening and diagnosis. First edition. Principles of evidence assessment and methods for reaching recommendations. Endoscopy 2012; 44: SE9-SE14

80 Moore RA, Derry S, McQuay HJ. Faecal blood loss with aspirin, nonsteroidal anti-inflammatory drugs and cyclo-oxygenase- 2 selective inhibitors: systematic review of randomized trials using autologous chromium-labelled erythrocytes. Arthritis Res Ther 2008; 10: R7

81 Morikawa T, Kato J, Yamaji Y et al. Sensitivity of immunochemical fecal occult blood test to small colorectal adenomas. Am J Gastroenterol 2007; 102: $2259-2264$

82 Morikawa T, Kato J, Yamaji Yet al. A comparison of the immunochemical fecal occult blood test and total colonoscopy in the asymptomatic population. Gastroenterology 2005; 129: $422-428$ 
83 Moslein G, Schneider C, Theilmeier A et al. [Analysis of the statistical value of various commercially available stool tests - a comparison of one stool sample in correlation to colonoscopy]. Dtsch Med Wochenschr 2010; 135: 557-562

84 Moss S, Ancelle-Park R, Brenner $H$. European guidelines for quality assurance in colorectal cancer screening and diagnosis. First edition. Evaluation and interpretation of screening outcomes. Endoscopy 2012; 44: SE49-SE64

85 Nakama H, Yamamoto $M$, Kamijo $\mathrm{N}$ et al. Colonoscopic evaluation of immunochemical fecal occult blood test for detection of colorectal neoplasia. Hepatogastroenterology 1999; 46: 228 -231

86 Nakama H, Zhang B, Fattah AS. A cost-effective analysis of the optimum number of stool specimens collected for immunochemical occult blood screening for colorectal cancer. Eur J Cancer 2000; 36: $647-650$

87 Nakama $H$, Zhang B, Zhang X. Evaluation of the optimum cut-off point in immunochemical occult blood testing in screening for colorectal cancer. Eur J Cancer 2001; 37: 398-401

88 Parekh M, Fendrick AM, Ladabaum U. As tests evolve and costs of cancer care rise: reappraising stool-based screening for colorectal neoplasia. Aliment Pharmacol Ther 2008; 27: 697-712

89 Pearson S, Bennitt W, Halloran S. Evaluation of eleven faecal occult blood test kits. London: Medical Devices Agency; 2000: MDA/2000/ 05

90 Pignone M, Campbell MK, Carr C et al. Meta-analysis of dietary restriction during fecal occult blood testing. Eff Clin Pract 2001; 4: 150-156

91 Piper MA. Immunochemical versus guaiac fecal occult blood tests. Chicago. IL, USA: Blue Cross and Blue Shield Association, Technology Evaluation Center. TEC Assessment Programs; 2004; 19 (5)

92 Quirke P, Risio M, Lambert R et al. European guidelines for quality assurance in colorectal cancer screening and diagnosis. First edition. Quality assurance in pathology in colorectal cancer screening and diagnosis. Endoscopy 2012; 44: SE116 - SE130

93 Ransohoff DF, Sandler RS. Clinical practice. Screening for colorectal cancer. N Engl J Med 2002; 346: 40-44

94 Rockey DC, Koch J, Cello JP et al. Relative frequency of upper gastrointestinal and colonic lesions in patients with positive fecal occultblood tests. N Engl J Med 1998; 339: 153-159

95 Rosenfield RE, Kochwa S, Kaczera $Z$ et al. Nonuniform distribution of occult blood in feces. Am J Clin Pathol 1979; 71: 204-209

96 Rozen P, Knaani J, Samuel Z. Performance characteristics and comparison of two immunochemical and two guaiac fecal occult blood screening tests for colorectal neoplasia. Dig Dis Sci 1997; 42: 20642071

97 Rozen P, Knaani J, Samuel Z. Eliminating the need for dietary restrictions when using a sensitive guaiac fecal occult blood test. Dig Dis Sci 1999; 44: 756-760

98 Rozen P, Knaani J, Samuel Z. Comparative screening with a sensitive guaiac and specific immunochemical occult blood test in an endoscopic study. Cancer 2000; 89: 46-52

99 Rozen P, Waked A, Vilkin A et al. Evaluation of a desk top instrument for the automated development and immunochemical quantification of fecal occult blood. Med Sci Monit 2006; 12: MT27-MT32

100 Rubeca T, Rapi S, Confortini $M$ et al. Evaluation of diagnostic accuracy of screening by fecal occult blood testing (FOBT). Comparison of FOB Gold and OC Sensor assays in a consecutive prospective screening series 70. Int J Biol Markers 2006; 21: 157-161

101 Said $R$. Contamination of urine with povidone-iodine. Cause of falsepositive test for occult blood in urine. JAMA 1979; 242: 748-749

102 Saito $H$. Screening for colorectal cancer by immunochemical fecal occult blood testing. Jpn J Cancer Res 1996; 87: 1011-1024

103 Saito H. Current status of colorectal cancer screening in Japan. Acta Endoscopica 2007; 37: 181 - 188

104 Saito H, Soma Y, Nakajima $M$ et al. A case-control study evaluating occult blood screening for colorectal cancer with hemoccult test and an immunochemical hemagglutination test. Oncol Rep 2000; 7: 815819

105 Schwartz MK. How do we detect hereditary large bowel cancer? Biochemical diagnosis Prog Clin Biol Res 1983; 115: 123 - 129

106 Scriven AJ, Tapley EM. Coloscreen VPI test kit evaluated for detection of fecal occult blood. Clin Chem 1989; 35: 156-158

107 European guidelines for quality assurance in colorectal cancer screening and diagnosis. First edition. Segnan N, Patnick J, von Karsa L. Luxembourg: European Commission, Publications Office of the European Union; 2010
108 Shastri YM, Naumann M, Oremek GM et al. Prospective multicenter evaluation of fecal tumor pyruvate kinase type M2 (M2-PK) as a screening biomarker for colorectal neoplasia. Int J Cancer 2006; 119: $2651-2656$

109 Sieg A, Thoms C, Luthgens K et al. Detection of colorectal neoplasms by the highly sensitive hemoglobin-haptoglobin complex in feces. Int J Colorectal Dis 1999; 14: 267-271

110 Sinatra MA, St John DJ, Young GP. Interference of plant peroxidases with guaiac-based fecal occult blood tests is avoidable. Clin Chem 1999; 45: $123-126$

111 Smith A, Young GP, Cole SR et al. Comparison of a brush-sampling fecal immunochemical test for hemoglobin with a sensitive guaiac-based fecal occult blood test in detection of colorectal neoplasia. Cancer 2006; 107: $2152-2159$

112 St John DJ, Young GP, Alexeyeff MA et al. Evaluation of new occult blood tests for detection of colorectal neoplasia. Gastroenterology 1993; 104: $1661-1668$

113 Starkey BJ. Screening for colorectal cancer. Ann Clin Biochem 2002; 39: $351-365$

114 Steele RJC, Pox C, Kuipers EJ et al. European guidelines for quality assurance in colorectal cancer screening and diagnosis. First edition. Managment of lesions detected in colorectal cancer screening. Endoscopy 2012; 44: SE140 - SE150

115 Steele RJC, Rey J-F, Lambert R. European guidelines for quality assurance in colorectal cancer screening and diagnosis. First edition. Professional requirements and training. Endoscopy 2012; 44: SE106SE115

116 Syed AA, Khatoon BA, Silwadi MF. New reagents for detection of faecal occult blood. J Pharm Biomed Anal 2001; 24: 581-586

117 Results of the first round of a demonstration pilot of screening for colorectal cancer in the United Kingdom. BMJ 2004; 329: 133

118 Valori R, Rey J-F, Atkin W et al. European guidelines for quality assurance in colorectal cancer screening and diagnosis. First edition. Quality assurance in endoscopy in colorectal screening and diagnosis. Endoscopy 2012; 44: SE88-SE105

119 van Roon AHC, Wilschut JA, van Ballegooijen $M$ et al. Attendance and diagnostic yield of 1 vs 2-sample fecal immunochemical test (FIT) screening: a comparative population-based colorectal cancer trial. Oral Presentation, Diagnostic Diseases Week 2010, New Orleans. 2010

120 van Rossum LG, van Rijn AF, Laheij RJ et al. Cutoff value determines the performance of a semi-quantitative immunochemical faecal occult blood test in a colorectal cancer screening programme. $\mathrm{Br} \mathrm{J}$ Cancer 2009; 101: $1274-1281$

121 van Rossum LG, van Rijn AF, Laheij RJ et al. Random comparison of guaiac and immunochemical fecal occult blood tests for colorectal cancer in a screening population. Gastroenterology 2008; 135: 82 90

122 Vilkin A, Rozen P, Levi Z et al. Performance characteristics and evaluation of an automated-developed and quantitative, immunochemical, fecal occult blood screening test. Am J Gastroenterol 2005; 100 : $2519-2525$

123 von Karsa L, Anttila A, Ronco G et al. Cancer Screening in the European Union. Report on the implementation of the Council Recommendation on Cancer Screening - First Report. Luxembourg: European Commission; 2008: http://ec.europa.eu/health/archive/ph_determinants/genetics/documents/cancer_screening.pdf Accessed 11/2/2012

124 von Karsa L, Lignini TA, Patnick J et al. The dimensions of the CRC problem. Best Pract Res Clin Gastroenterol 2010; 24: 381-396

125 von Roon AC, Karamountzos L, Purkayastha $S$ et al. Diagnostic precision of fecal calprotectin for inflammatory bowel disease and colorectal malignancy. Am J Gastroenterol 2007; 102: 803-813

126 Wang DR, Tang $D$. Hypermethylated SFRP2 gene in fecal DNA is a high potential biomarker for colorectal cancer noninvasive screening. World J Gastroenterol 2008; 14: 524-531

127 Welch CL, Young DS. Spectrophotometry of occult blood in feces. Clin Chem 1983; 29: 2022-2025

128 Wilson JM, Jungner YG. Principles and practice of mass screening for disease. World Health Organization; 1968: http://whqlibdoc.who. int/php/WHO_PHP_34.pdf Accessed 11/2/2012

129 Wong $B C$, Wong WM, Cheung KL et al. A sensitive guaiac faecal occult blood test is less useful than an immunochemical test for colorectal cancer screening in a Chinese population. Aliment Pharmacol Ther 2003; 18: 941 -946 
130 Young GP, Cole S. New stool screening tests for colorectal cancer. Digestion 2007; 76: 26-33

131 Young GP, Sinatra MA, St John DJ. Influence of delay in stool sampling on fecal occult blood test sensitivity. Clin Chem 1996; 42: 1107-1108

132 Young GP, St John DJ, Cole SR et al. Prescreening evaluation of a brushbased faecal immunochemical test for haemoglobin. J Med Screen 2003; 10: $123-128$

133 Young GP, St John DJ, Winawer SJ et al. Choice of fecal occult blood tests for colorectal cancer screening: recommendations based on performance characteristics in population studies: a WHO (World Health Organization) and OMED (World Organization for Digestive Endoscopy) report. Am J Gastroenterol 2002; 97: 2499-2507
134 Zappa M, Castiglione G, Paci E et al. Measuring interval cancers in population-based screening using different assays of fecal occult blood testing: the District of Florence experience. Int J Cancer 2001; 92: $151-154$

135 Zappa M, Visioli CB, Ciatto $S$ et al. Gastric cancer after positive screening faecal occult blood testing and negative assessment. Dig Liver Dis 2007; 39: 321 - 326

136 Zhou L, Yu H, Zheng S. [The value of "occult blood bead" in detection of upper digestive tract disorders with bleeding]. Zhonghua Zhong Liu Za Zhi 1999; 21: $48-50$

137 Zorzi M, Falcini F, Fedato $C$ et al. Screening for colorectal cancer in Italy: 2006 survey. Epidemiol Prev 2008; 32: 55-68 